PHYSICAL REVIEW D 89, 114016 (2014)

\title{
Prompt-photon plus jet associated photoproduction at HERA in the parton Reggeization approach
}

\author{
B. A. Kniehl" \\ II. Institut für Theoretische Physik, Universität Hamburg, \\ Luruper Chaussee 149, 22761 Hamburg, Germany \\ M. A. Nefedov ${ }^{\dagger}$ \\ Samara State University, Academician Pavlov Street 1, 443011 Samara, Russia \\ and II. Institut für Theoretische Physik, Universität Hamburg, \\ Luruper Chaussee 149, 22761 Hamburg, Germany \\ V. A. Saleev \\ Samara State University, Academician Pavlov Street 1, 443011 Samara, Russia \\ and S. P. Korolyov Samara State Aerospace University, Moscow Highway 34, 443086 Samara, Russia
} (Received 14 April 2014; published 17 June 2014)

\begin{abstract}
We study the photoproduction of isolated prompt photons associated with hadron jets in the framework of the parton Reggeization approach. The cross section distributions in the transverse energies and pseudorapidities of the prompt photon and the jet as well as the azimuthal-decorrelation variables measured by the H1 and ZEUS collaborations at DESY HERA are nicely described by our predictions. The main improvements with respect to previous studies in the $k_{T}$-factorization framework include the application of the Reggeized-quark formalism, the generation of exactly gauge-invariant amplitudes with off-shell initialstate quarks, and the exact treatment of the $\gamma R \rightarrow \gamma g$ box contribution with off-shell initial-state gluons.

DOI: 10.1103/PhysRevD.89.114016

PACS numbers: 12.38.Bx, 12.39.St, 12.40.Nn, 13.87.Ce
\end{abstract}

\section{INTRODUCTION}

The photoproduction of prompt photons with large transverse momenta provides a formidable laboratory for precision tests of perturbative quantum chromodynamics (QCD) and a useful source of information on the parton content of the proton and the real photon. The initial-state photon may interact with the partons inside the proton either directly (direct photoproduction) or via its partonic content (resolved photoproduction).

The inclusive photoproduction of prompt photons, singly and in association with jets, received a lot of attention, both experimentally and theoretically. On the experimental side, the H1 [1,2] and ZEUS [3-5] collaborations measured the cross section distributions in the transverse energies $\left(E_{T}\right)$ and the pseudorapidities $(\eta)$ of the prompt photon and the jet as well as in azimuthal-decorrelation parameters such as the azimuthal angle enclosed between the prompt-photon and jet transverse momenta $(\Delta \phi)$ and the component of the prompt-photon transverse momentum orthogonal to the direction of the jet transverse momentum $\left(p_{\perp}\right)$. Also, the distributions in the variables estimating the momentum fractions of the initial-state partons $x_{p}^{\mathrm{LO}}, x_{\gamma}^{\mathrm{LO}}$, and $x_{\gamma}^{\mathrm{obs}}$ were measured. This rich set of observables allows one to perform a detailed study of the underlying partonic

\footnotetext{
*kniehl@desy.de nefedovma@gmail.com

¥saleev@samsu.ru
}

processes and to assess the relevance of different perturbative corrections.

On the theoretical side, attempts to describe these data were made both at next-to-leading order (NLO) in the conventional collinear parton model (CPM) $[6,7]$ and in approaches accommodating off-shell initial-state partons, such as the $k_{T}$-factorization approach (KFA) [8-10] and its implementation with Reggeized partons, which we refer to as the parton Reggeization approach (PRA) [11]. In the case of inclusive prompt-photon photoproduction, both the NLO CPM and leading-order (LO) KFA predictions underestimate all the measured distributions, as may be seen, e.g., from the comparative figures in Refs. [1,2,4], while the LO PRA predictions describe the $E_{T}$ distributions quite well and the $\eta$ distributions reasonably well [11].

As for prompt-photon plus jet associated photoproduction, NLO CPM predictions generally agree with the measured $\eta$ distributions, slightly underestimate the $E_{T}$ distributions, and provide a poor description of the azimuthal-decorrelation observables [1,2], due to the fact that these distributions collapse to delta functions at LO in the CPM and, therefore, strongly depend on the radiation of additional partons. The available KFA predictions provide a better description of the measured $E_{T}$ distributions and azimuthal-decorrelation observables, but are implemented with matrix elements that manifestly violate gauge invariance, which renders the quantitative improvements of the predictions questionable. Furthermore, in the early studies 
$[8,9]$, the partonic subprocess pertaining to the scattering of a photon and an off-shell gluon $\gamma g^{*} \rightarrow \gamma g$ was not taken into account. Later, this contribution was found to be numerically significant [10], due to the large gluon luminosity under HERA conditions. But the treatment of this contribution was approximate because the virtuality of the initial-state gluon was not taken into account at the amplitude level, but only in the kinematics of the process [10].

In view of the shortcomings of the previous calculations mentioned above, it is an urgent matter to perform an improved analysis of prompt-photon plus jet associated photoproduction in the PRA, which allows one to treat offshell initial-state quarks and gluons in a gauge-invariant way. Moreover, it is crucial to include the full dependence on the transverse momentum of the off-shell (Reggeized) initial-state gluon $R$ in the process $\gamma R \rightarrow \gamma g$. These are two main goals of the present paper.

This paper has the following structure. In Sec. II, a basic introduction to the PRA, a list of the relevant partonic subprocesses, and the amplitudes for the tree-level contributions are presented. In Sec. III, the calculation of the one-loop amplitude of the partonic subprocess $\gamma R \rightarrow \gamma g$ is discussed in some detail, and the cross-checks applied to the results obtained are described. A compact expression of this amplitude is presented in the Appendix. The results of the numerical calculations and comparisons with experimental data and previous studies are carefully discussed in Sec. IV, and a few concluding remarks are collected in Sec. V.

\section{PRA FORMALISM AND TREE-LEVEL CONTRIBUTIONS}

In hadron-hadron or lepton-hadron collisions with large center-of-mass energies $\sqrt{S}$, different kinds of perturbative corrections are relevant for different processes and different regions of phase space. For example, the higher-order corrections for the production of heavy final states, such as Higgs bosons, top-quark pairs, dijets with large invariant masses, or Drell-Yan pairs, by initial-state partons with relatively large momentum fractions $x \sim 10^{-1}$ are dominated by soft and collinear gluons and may increase the cross sections up to a factor of 2 .

By contrast, relatively light final states, such as smalltransverse-momentum heavy quarkonia, single jets, prompt photons, or dijets with small invariant masses, are produced by the fusion of partons with small values of $x$, typically $x \sim 10^{-3}$, because of the large values of $\sqrt{S}$. Radiative corrections to such processes are dominated by the production of additional hard jets. The only way to treat such processes in the conventional CPM is to calculate higherorder corrections in the strong coupling constant $\alpha_{s}=$ $g_{s}^{2} /(4 \pi)$, which could be a challenging task for some processes even at the NLO level. To overcome this difficulty and take into account a sizable part of the higher-order corrections in the small- $x$ regime, the KFA, also known as high-energy factorization approach, was introduced [12]. The KFA works with off-shell initial-state partons, which carry not only a fraction $x$ of longitudinal momentum, but also a significant transverse momentum $\mathbf{q}_{T}$, with $\left|\mathbf{q}_{T}\right| \sim x \sqrt{S}$. The corresponding factorization formula may be schematically represented as

$$
\begin{aligned}
d \sigma(\mathcal{Y})= & \sum_{i, j} \Phi_{i}\left(x_{1}, t_{1}, \mu_{F}\right) \otimes \Phi_{j}\left(x_{2}, t_{2}, \mu_{F}\right) \\
& \otimes d \hat{\sigma}_{i j}\left(x_{1}, \mathbf{q}_{T 1}, x_{2}, \mathbf{q}_{T 2}, \mathcal{Y}\right),
\end{aligned}
$$

where the sum runs over the parton species $i, j, \otimes$ denotes a convolution over the relevant momentum components of the partons, $\mathcal{Y}$ is the set of kinematic variables of the final state, and $d \hat{\sigma}_{i j}$ are the partonic cross sections. The unintegrated parton distribution function (unPDF) $\Phi_{i}\left(x_{i}, t_{i}, \mu_{F}\right)$ depends on the longitudinal-momentum fraction $x_{i}$ and the virtuality $t_{i}=\mathbf{q}_{T i}^{2}$ of the parton and the factorization scale $\mu_{F}$, which separates the stages of the evolution of the unPDF and the hard scattering. The unPDF is normalized by the following condition:

$$
\int^{\mu_{F}^{2}} d t \Phi_{i}\left(x, t, \mu_{F}\right)=x f_{i}\left(x, \mu_{F}\right),
$$

where $f_{i}\left(x, \mu_{F}\right)$ is the respective CPM PDF.

In the asymptotic high-energy (Regge) regime, the characteristic scales of the scattering process obey the following hierarchy: $\Lambda_{\mathrm{QCD}} \ll \mu_{F} \sim \mu_{R} \ll \sqrt{S}$, where $\Lambda_{\mathrm{QCD}}$ is the asymptotic scale parameter of QCD and $\mu_{R}$ is the renormalization scale. Deeply in the Regge regime, all the produced particles are highly separated in rapidity, obeying the so-called multi-Regge kinematics, while the $k_{T}$ ordering of the Dokshitzer-Gribov-Lipatov-Altarelli-Parisi (DGLAP) [13] evolution is completely broken. So, the evolution of the unPDFs is governed by large logarithms of a new type, namely $\log (1 / x)$. To resum these logarithms, the Balitsky-Fadin-Kuraev-Lipatov (BFKL) evolution equation was introduced [14]. This leads to a powerlike growth of the gluon unPDF in the small- $x$ region, while the effect on the quark unPDFs is subleading, so that the gluon unPDF is expected to strongly dominate at high energies.

At intermediate energies, however, the quark unPDFs and DGLAP effects cannot be neglected, and the unPDFs may be obtained by certain approaches taking into account both DGLAP and BFKL effects, for example by the Kimber-Martin-Ryskin approach [15] or by a solution to the Ciafaloni-Catani-Fiorani-Marchesini evolution equation [16].

Special care is required to define hard-scattering matrix elements in the KFA because initial-state partons are now off shell, which generally entails gauge dependence in QCD. In the KFA studies of heavy-quark pair production or deep-inelastic scattering, the polarization vector of the initial-state gluon with four-momentum $k^{\mu}=\left(k_{0}, \mathbf{k}_{T}, k_{z}\right)$ is usually taken to be 


$$
\varepsilon^{\mu}(k)=\frac{k_{T}^{\mu}}{\left|\mathbf{k}_{T}\right|},
$$

where $k_{T}^{\mu}=\left(0, \mathbf{k}_{T}, 0\right)$, in analogy with the equivalent-photon approximation in QED [12]. However, this prescription does not lead to gauge-invariant results for hard-scattering amplitudes with gluons in the final state because of their involved non-Abelian color structure. Furthermore, the usual KFA does not provide a generally accepted prescription for the treatment of off-shell initial-state quarks.

A rigorous way to solve this gauge-dependence problem is to observe that the small- $x$ regime, with $x \sim \mu_{F} / \sqrt{S} \ll 1$, implies that particles produced in the hard interaction are strongly separated in rapidity from the particles produced at the unPDF evolution stage. The regime where the produced particles are grouped in a few clusters which are strongly separated in rapidity is characterized by what is called quasi-multi-Regge kinematics (QMRK). It was shown $[17,18]$ that, in the QMRK, the gauge-invariance conditions hold for each cluster separately and that the fields carrying four-momentum between these clusters are new gaugeinvariant degrees of freedom accompanying the usual Yang-Mills gluons and quarks in the effective field theory for the Regge limit of QCD [19], the Reggeized gluons [19] and quarks [20]. The implementation of the KFA characterized by Eq. (1) with partonic cross sections obtained using the Feynman rules of the effective field theory for the Regge limit of QCD $[20,21]$ is referred to as the PRA.

The hard-scattering amplitudes in the PRA coincide with those obtained using the prescription in Eq. (3) whenever the application of the latter is safe, as was explicitly shown, e.g., for heavy-quark [22] and heavy-quarkonium production [23,24]. Recent examples of nontrivial applications of the PRA to high-energy phenomenology include the description of dijet azimuthal decorrelations [25] as well as the production of bottom-flavored jets [26], Drell-Yan lepton pairs [27], single jets, and prompt photons [28] at the Tevatron and the LHC.

We now turn from the general discussion of the relationship between the PRA and the KFA to the application of the PRA to prompt-photon plus jet associated photoproduction. The LO QMRK approximation for this process corresponds to only including $2 \rightarrow 2$ subprocesses yielding potentially sizable contributions. These partonic subprocesses may be classified into direct-photoproduction ones, where the photon directly takes part in the hard scattering, and resolved-photoproduction ones, in which the photon interacts as a composite object containing quarks and gluons. The LO direct-photoproduction subprocesses are

$$
\begin{aligned}
& Q\left(q_{1}\right)+\gamma\left(q_{2}\right) \rightarrow q\left(q_{3}\right)+\gamma\left(q_{4}\right), \\
& R\left(q_{1}\right)+\gamma\left(q_{2}\right) \rightarrow g\left(q_{3}\right)+\gamma\left(q_{4}\right),
\end{aligned}
$$

where $Q$ and $R$ are the Reggeized quark and gluon from the proton and the four-momenta of the partons are given in parentheses. Here, the charge-conjugated subprocesses involving the Reggeized antiquark $\bar{Q}$ are also implied. The contribution to the cross section of the partonic subprocess in Eq. (4) is of order $\mathcal{O}\left(\alpha^{2}\right)$, where $\alpha$ is Sommerfeld's fine-structure constant. The contribution from the partonic subprocess in Eq. (5) is formally of order $\mathcal{O}\left(\alpha^{2} \alpha_{s}^{2}\right)$. However, due to the large values of the gluon unPDF at small values of $x$, this process should be taken into account already at LO in the PRA. The LO resolved-photoproduction subprocesses are

$$
\begin{aligned}
& R\left(q_{1}\right)+q[\gamma]\left(\tilde{q}_{2}\right) \rightarrow q\left(q_{3}\right)+\gamma\left(q_{4}\right), \\
& Q\left(q_{1}\right)+\bar{q}[\gamma]\left(\tilde{q}_{2}\right) \rightarrow g\left(q_{3}\right)+\gamma\left(q_{4}\right), \\
& Q\left(q_{1}\right)+g[\gamma]\left(\tilde{q}_{2}\right) \rightarrow q\left(q_{3}\right)+\gamma\left(q_{4}\right),
\end{aligned}
$$

and their charge-conjugated counterparts. The partonic subprocess in Eq. (6) is important because of the abovementioned amplification by the gluon unPDF of the proton. By detailed inspection, we find the partonic subprocesses in Eqs. (7) and (8) to account for less than 5\% of the total cross section and omit their contributions in the following.

In addition, partonic subprocesses in which finalstate partons fragment to photons should be considered. However, their contribution is strongly suppressed by the photon-isolation condition applied to the experimental data, which constrains the hadronic energy within the photonisolation cone to be less than $10 \%$ of the photon energy. In other words, more than $90 \%$ of the parton energy must be transmitted to the photon, which rarely happens. We explicitly verify the strong suppression of the fragmentation contributions, which was also observed in Ref. [11].

All the $2 \rightarrow 3$ subprocesses contribute at NLO in the PRA. In order to avoid double counting of contributions to unPDFs and hard-scattering matrix elements due to the emission of additional partons, one may impose the condition that there are no rapidity gaps between unobserved and observed partons, which requires a proper subtraction procedure, as described in Ref. [29]. Contributions of this type and from the interference of one-loop and tree-level $2 \rightarrow 2$ scattering amplitudes constitute nonfactorizable higher-order corrections in our approach, in contrast to those which can be factorized into unPDFs. In the present paper, we focus on the LO contributions.

In the remainder of this section, we outline the derivation of the amplitudes for the tree-level subprocesses in Eqs. (4) and (6). We start by introducing the basic kinematic notation to be used throughout this paper. We work in the laboratory frame and take the $z$ axis to point along the flight direction of the proton, whose mass we neglect. It is convenient to introduce the light-cone four-vectors

$$
n_{+}^{\mu}=\frac{P_{2}^{\mu}}{E_{2}}, \quad n_{-}^{\mu}=\frac{P_{1}^{\mu}}{E_{1}}
$$




\section{B. A. KNIEHL, M. A. NEFEDOV, AND V. A. SALEEV}

where $P_{1}$ and $P_{2}$ are the four-momenta of the proton and the electron, respectively, and $E_{1}$ and $E_{2}$ are their energies. We have $n_{ \pm}^{2}=0$ and $n_{+} \cdot n_{-}=2$. Then, any four-vector $k^{\mu}$ may be expressed in terms of its light-cone components, $k^{ \pm}=n_{ \pm} \cdot k=k^{0} \pm k^{3}$, as

$$
k^{\mu}=\frac{1}{2}\left(k^{+} n_{-}^{\mu}+k^{-} n_{+}^{\mu}\right)+k_{T}^{\mu},
$$

and we have $k_{T} \cdot n_{ \pm}=0$. The four-momentum of the Reggeized parton from the proton can be written as $q_{1}=$ $x_{1} P_{1}+q_{T 1}$ and has virtuality $q_{1}^{2}=q_{T 1}^{2}=-\mathbf{q}_{T 1}^{2}=-t_{1}$. The quasireal photon carries the fraction $y$ of the electron energy and has four-momentum $q_{2}=y P_{2}$. If the photon is resolved, then it transfers the fraction $x_{2}$ of its energy to the offspring parton, which has four-momentum $\tilde{q}_{2}=x_{2} q_{2}=x_{2} y P_{2}$. In the following, we assume the photon to be direct; the resolved-photon results are recovered by replacing $q_{2}$ with $\tilde{q}_{2}$. The square of the proton-photon center-of-mass energy is $S=2 P_{1} \cdot q_{2}=4 y E_{1} E_{2}$. The partonic Mandelstam variables are defined as

$$
s=\left(q_{3}+q_{4}\right)^{2}, \quad t=\left(q_{2}-q_{4}\right)^{2}, \quad u=\left(q_{2}-q_{3}\right)^{2},
$$

where $q_{3}$ and $q_{4}$ are the four-momenta of the final-state particles, which we take to be massless, and we have $s+t+u=-t_{1}$. They may be expressed in terms of the final-state light-cone four-momenta as

$s=\left(q_{3}^{+}+q_{4}^{+}\right)\left(q_{3}^{-}+q_{4}^{-}\right)-t_{1}, \quad t=-q_{4}^{+}\left(q_{3}^{-}+q_{4}^{-}\right)$,

$u=-q_{3}^{+}\left(q_{3}^{-}+q_{4}^{-}\right)$.

It turns out that the hard-scattering amplitudes may be cast into a particularly compact form by using the dimensionless Sudakov variables instead of the light-cone ones. They are defined as

$$
\begin{aligned}
& a_{3,4}=\frac{2 q_{2} \cdot q_{3,4}}{S}=\frac{2 y E_{2} q_{3,4}^{+}}{S}, \\
& b_{3,4}=\frac{2 P_{1} \cdot q_{3,4}}{S}=\frac{2 E_{1} q_{3,4}^{-}}{S},
\end{aligned}
$$

so that $a_{3}+a_{4}=x_{1}$ and $b_{3}+b_{4}=1$, or $b_{3}+b_{4}=x_{2}$ in the resolved-photon case.

In addition to the standard Feynman rules of QCD, we need the couplings of the Reggeized quarks and gluons to the ordinary quarks, gluons, and photons. The full list of the latter may be found in Refs. [20,21]. For the reader's convenience, we specify the Feynman rules relevant for our calculation in Fig. 1.

The Feynman diagrams contributing at LO to the partonic subprocess in Eq. (4) are shown in Fig. 2. Using the Feynman rules in Fig. 1 and the light-cone four-vectors defined above, we find the modulus square of the hard-scattering amplitude averaged over the spins and
PHYSICAL REVIEW D 89, 114016 (2014)

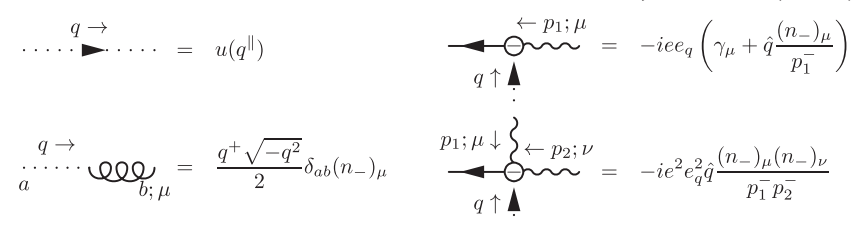

FIG. 1. Feynman rules for the Reggeized quark and gluon in the initial state (left panel) and for the $Q \gamma q$ and $Q \gamma \gamma q$ vertices (right panel).
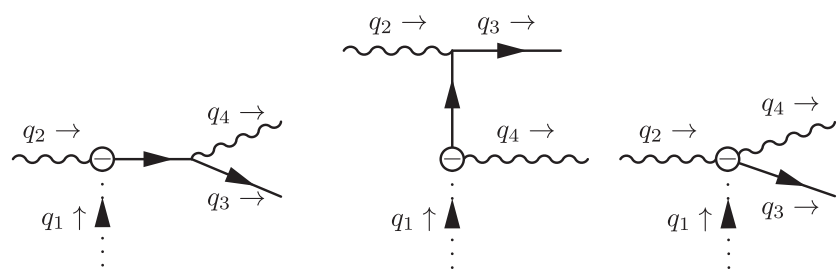

FIG. 2. Feynman diagrams contributing at LO to the partonic subprocess in Eq. (4).

colors of the incoming partons and summed over those of the outgoing ones to be

$$
\overline{|\mathcal{M}(Q \gamma \rightarrow q \gamma)|^{2}}=-32 \pi^{2} \alpha^{2} e_{q}^{4} \frac{S x_{1}}{b_{4} s u}\left(t_{1} b_{3}^{3}+s b_{4}^{3}-u\right),
$$

where $e_{q}$ is the quark electric charge in the units of the positron charge. In the limit when the initial-state Reggeized quark goes on shell, which amounts to substituting $t_{1} \rightarrow 0, a_{3} \rightarrow-u / S, a_{4} \rightarrow-t / S, b_{3} \rightarrow-t /\left(x_{1} S\right)$, $b_{4} \rightarrow-u /\left(x_{1} S\right)$, and $x_{1} \rightarrow s / S$, Eq. (14) reproduces the well-known LO CPM result for Compton scattering,

$$
\overline{|\mathcal{M}(q \gamma \rightarrow q \gamma)|^{2}}=-32 \pi^{2} \alpha^{2} e_{q}^{4}\left(\frac{s}{u}+\frac{u}{s}\right) .
$$

The Feynman diagrams contributing to the partonic subprocess in Eq. (6) are depicted in Fig. 3. The modulus square of the hard-scattering amplitude averaged over the spins and colors in the initial state and summed over those in the final state reads

$$
\begin{aligned}
\overline{|\mathcal{M}(R q \rightarrow q \gamma)|^{2}}= & \frac{16}{3} \pi^{2} \alpha \alpha_{s} e_{q}^{2} \frac{S^{2} x_{1}^{2} x_{2}}{s t^{2} t_{1}}\left\{t \left[u b_{3}+(t+u) b_{4}\right.\right. \\
& \left.-S a_{3} b_{3}^{2}+s x_{2}\right]+S a_{4} b_{3}\left[s b_{4}-t b_{3}\right. \\
& \left.\left.-(s+t) x_{2}\right]\right\} .
\end{aligned}
$$

The CPM limit of Eq. (16) is defined as

$$
\lim _{t_{1} \rightarrow 0} \int_{0}^{2 \pi} \frac{d \phi_{1}}{2 \pi} \overline{|\mathcal{M}(R q \rightarrow q \gamma)|^{2}}=\overline{|\mathcal{M}(g q \rightarrow q \gamma)|^{2}},
$$

where $\phi_{1}$ is the azimuthal angle enclosed between the three-vectors $\mathbf{q}_{T 1}$ and $\mathbf{q}_{T 3}$. Note that the order of integrating 


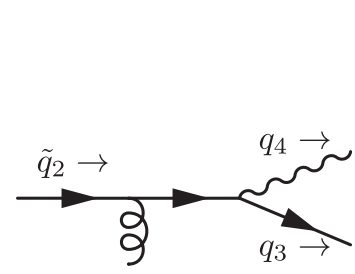

$q_{1} \uparrow:$

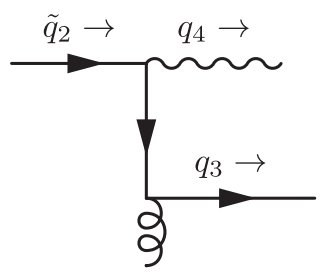

$q_{1} \uparrow:$
FIG. 3. Feynman diagrams contributing at LO to the partonic subprocess in Eq. (6).

and taking the limit may be safely reversed in Eq. (17). The limit $t_{1} \rightarrow 0$ may be taken in Eq. (16) by substituting $a_{3} \rightarrow-u /\left(S x_{2}\right), a_{4} \rightarrow-t /\left(S x_{2}\right), b_{3} \rightarrow\left(-t+B \sqrt{t_{1}}\right) /\left(S x_{1}\right)$, $b_{4} \rightarrow\left(-u-B \sqrt{t_{1}}\right) /\left(S x_{1}\right)$, and $S x_{1} x_{2} \rightarrow s+t_{1}$, where $B=\sqrt{2 u t / s} \cos \phi_{1}$. We thus recover the well-known LO CPM result

$$
\overline{|\mathcal{M}(g q \rightarrow q \gamma)|^{2}}=-\frac{16}{3} \pi^{2} \alpha \alpha_{s} e_{q}^{2}\left(\frac{s}{t}+\frac{t}{s}\right)
$$

Recently, an alternative method, which is equivalent to the PRA involving Reggeized quarks and gluons adopted here, was proposed in Ref. [30]. It amounts to embedding the $2 \rightarrow n$ scattering processes under consideration here into auxiliary $2 \rightarrow n+2$ scattering processes and to extracting from them the gauge-invariant $2 \rightarrow n$ amplitudes with off-shell initial-state partons by using the spinorhelicity representation with complex momenta. This is more suitable for the implementation in automatic matrix-element generators, but the use of Reggeized quarks and gluons is by far simpler for the purposes of the present study.

\section{BOX CONTRIBUTION}

In this section, we discuss the hard-scattering amplitude of the one-loop subprocess in Eq. (5) within the PRA. Specifically, we derive the helicity amplitudes and verify that they reproduce the well-known expressions for photonby-photon scattering [31] in the CPM limit.

The contributing Feynman diagrams are shown in Fig. 4. Using the Feynman rules in Fig. 1, we may write the helicity amplitudes as

$$
\begin{aligned}
\mathcal{M}\left(R \lambda_{2}, \lambda_{3} \lambda_{4}\right)= & -\frac{q_{1}^{+}}{2 \sqrt{t_{1}}}\left(n_{-}\right)_{\mu_{1}} \varepsilon_{\mu_{2}}\left(1,-\lambda_{2}\right) \varepsilon_{\mu_{3}}^{*}\left(2, \lambda_{3}\right) \\
& \times \varepsilon_{\mu_{4}}^{*}\left(2,-\lambda_{4}\right) \mathcal{M}^{\mu_{1} \mu_{2} \mu_{3} \mu_{4}},
\end{aligned}
$$

where $\lambda_{i}= \pm 1$ are the helicities of the massless vector bosons and the overall factor

$$
\frac{(4 \pi)^{2} \alpha \alpha_{s}}{(2 \pi)^{4}} \frac{\delta_{a b}}{2}\left(\sum_{q} e_{q}^{2}\right)
$$

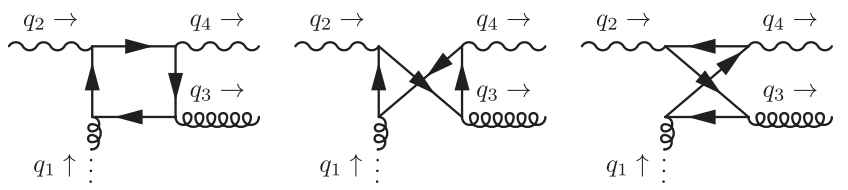

FIG. 4. Feynman diagrams contributing at LO to the partonic subprocess in Eq. (5). The diagrams with reversed fermionnumber flow are not shown.

has been omitted on the right-hand side of Eq. (19) for the ease of notation. The fourth-rank vacuum polarization tensor $\mathcal{M}^{\mu_{1} \mu_{2} \mu_{3} \mu_{4}}$ in Eq. (19) reads

$\mathcal{M}^{\mu_{1} \mu_{2} \mu_{3} \mu_{4}}$

$$
\begin{aligned}
& =2 \int d^{4} q\left\{\frac{\operatorname{tr}\left[\left(\hat{q}-\hat{q}_{1}\right) \gamma^{\mu_{3}}\left(\hat{q}+\hat{q}_{2}-\hat{q}_{4}\right) \gamma^{\mu_{4}}\left(\hat{q}+\hat{q}_{2}\right) \gamma^{\mu_{2}} \hat{q} \gamma^{\mu_{1}}\right]}{\left(q-q_{1}\right)^{2}\left(q+q_{2}-q_{4}\right)^{2}\left(q+q_{2}\right)^{2} q^{2}}\right. \\
& \left.+\left(q_{3} \leftrightarrow q_{4}, \mu_{3} \leftrightarrow \mu_{4}\right)+\left(q_{4} \leftrightarrow-q_{2}, \mu_{4} \leftrightarrow \mu_{2}\right)\right\}
\end{aligned}
$$

where the overall factor of 2 accounts for the Feynman diagram with the fermion-number flow reversed. The polarization four-vectors in Eq. (19) read

$$
\varepsilon(j, \lambda)=\frac{1}{\sqrt{2}}\left(n_{x}^{(j)}+i \lambda n_{y}^{(j)}\right)
$$

where

$$
\begin{aligned}
n_{x}^{(1)} & =\frac{1}{\Delta}\left[\left(q_{2} \cdot q_{3}\right) q-\left(q \cdot q_{3}\right) q_{2}-\left(q \cdot q_{2}\right) q_{3}\right], \\
n_{x}^{(2)} & =\frac{1}{\Delta}\left[\left(q_{3} \cdot q_{4}\right) q-\left(q \cdot q_{4}\right) q_{3}-\left(q \cdot q_{3}\right) q_{4}\right], \\
\left(n_{y}^{(1)}\right)^{\mu} & =-\left(n_{y}^{(2)}\right)^{\mu}=\frac{1}{\Delta} \epsilon^{\mu q_{2} q_{3} q_{4}} \equiv n_{y}^{\mu},
\end{aligned}
$$

with $\Delta=\sqrt{s t u} / 2$ and $q=q_{2}+q_{3}$. The handling of the four-vector $n_{y}$ may be facilitated by observing that it has the scalar products

$$
q_{2} \cdot n_{y}=q_{3} \cdot n_{y}=q_{4} \cdot n_{y}=n_{+} \cdot n_{y}=0, \quad n_{y}^{2}=-1,
$$

and that the four-vector $n_{-}$appearing in Eq. (19) may be decomposed as

$$
n_{-}=\alpha n_{+}+\beta_{1} q_{3}+\beta_{2} q_{4}+\gamma n_{y},
$$

with the coefficients

$$
\begin{aligned}
\alpha & =\frac{s_{+}-s}{2 q_{3}^{+} q_{4}^{+}}, \quad \beta_{1}=\frac{s+s_{-}}{s q_{3}^{+}}, \quad \beta_{2}=\frac{s-s_{-}}{s q_{4}^{+}}, \\
\gamma & =\frac{2 y E_{2}}{\Delta}\left|\mathbf{q}_{T 3}\right|\left|\mathbf{q}_{T 4}\right| \sin (\Delta \phi),
\end{aligned}
$$


where $s_{ \pm}=q_{4}^{-} q_{3}^{+} \pm q_{4}^{+} q_{3}^{-}$and $\Delta \phi$ is the azimuthal angle enclosed between $\mathbf{q}_{T 3}$ and $\mathbf{q}_{T 4}$. Exploiting the fact that $n_{-}^{2}=0$, we may express $\gamma^{2}$ through light-cone components as

$$
\gamma^{2}=\frac{2 s s_{+}-s^{2}-s_{-}^{2}}{s q_{3}^{+} q_{4}^{+}} .
$$

In the CPM limit, the four-vectors $n_{+}, n_{-}, q_{3}$, and $q_{4}$ become linearly dependent, and $\gamma \rightarrow 0$. For the sake of a compact expression for Eq. (19), we introduce the variable

$$
\gamma_{1}=\frac{q_{3}^{+} \Delta}{\sqrt{t_{1}}} \gamma=\frac{u}{\sqrt{t_{1}}}\left|\mathbf{q}_{T 3}\right|\left|\mathbf{q}_{T 4}\right| \sin (\Delta \phi),
$$

which has a nonvanishing CPM limit,

$$
\gamma_{1} \rightarrow 2 \frac{u}{s} \Delta \sin \phi_{1}
$$

A similar variable $\gamma_{2}$ is related to the product

$$
q_{3}^{-} q_{3}^{+}=\frac{u}{(t+u)^{2}}\left[(u-t)\left(t_{1}-t\right)-2 t^{2}+\gamma_{2} \sqrt{t_{1}}\right] .
$$

It may be expressed through $\gamma_{1}$ using Eqs. (12), (26), and (27) as

$$
\gamma_{2}=2 \zeta \sqrt{s t u-\frac{(t+u)^{2}}{u^{2}} \gamma_{1}^{2}},
$$

where the sign factor $\zeta= \pm 1$ is to be determined so that the product of Eq. (29) with $t$ always coincides with $u q_{3}^{-} q_{4}^{+}$in compliance with Eq. (12). In the CPM limit, we have

$$
\gamma_{2} \rightarrow 4 \Delta \cos \phi_{1} .
$$

These new variables allow us to express Eq. (19) in a simple form that is manifestly finite in the CPM limit. All the dependences on the light-cone components reside in $\gamma_{1}$, while the residual parts of the expression depend only on the Mandelstam variables. The exact analytical expressions for all helicity amplitudes and the squared amplitude are presented in the Appendix in terms of the dimensionally regularized one-loop scalar integrals $B_{0}, C_{0}$, and $D_{0}$ defined as in Ref. [32]. The cancellations of the ultraviolet and infrared divergences are explicit in these expressions and are also checked in the numerical calculations.

Another important consistency check is to recover the well-known result in the CPM limit [31]. The relationship analogous to Eq. (17) may be written as

$$
\int_{0}^{2 \pi} \frac{d \phi_{1}}{2 \pi} \lim _{t_{1} \rightarrow 0} \overline{\left|\mathcal{M}\left(R \lambda_{2}, \lambda_{3} \lambda_{4}\right)\right|^{2}}=\frac{|N|^{2}}{2} \sum_{\lambda_{1}= \pm}\left|\mathcal{M}\left(\lambda_{1} \lambda_{2}, \lambda_{3} \lambda_{4}\right)\right|^{2},
$$

where the normalization factor $N=8 \pi^{2} i$ has been pulled out of the CPM amplitudes, so that

$$
\begin{aligned}
\mathcal{M}(++,+-) & =\mathcal{M}(++,-+)=\mathcal{M}(+-,++) \\
& =\mathcal{M}(-+,++)=\mathcal{M}(++,--)=-1 .
\end{aligned}
$$

In the following, we set $\lambda_{2}=+1$ without loss of generality. For $\lambda_{3}=\lambda_{4}=-1$, Eq. (32) may be immediately verified using Eqs. (28) and (A4). The other three combinations of $\lambda_{3}$ and $\lambda_{4}$ are slightly more involved.

Let us consider the case $\lambda_{3}=\lambda_{4}=+1$ as an example. We first recall that [31]

$$
\begin{aligned}
\mathcal{M}(++,++)= & 1+\frac{u-t}{s}\left[B_{0}(t)-B_{0}(u)\right]+\frac{t^{2}+u^{2}}{s^{2}} \\
& \times\left[t C_{0}(t)+u C_{0}(u)-\frac{t u}{2} D_{0}(t, u)\right],
\end{aligned}
$$

where the shorthand notation for the scalar one-loop integrals $B_{0}, C_{0}$, and $D_{0}$ is explained in the Appendix. On the other hand, substituting Eqs. (28) and (31) in Eq. (A1) and using $s+t+u=0$, we find

$$
\begin{aligned}
\lim _{t_{1} \rightarrow 0} \mathcal{M}(R+,++)= & 4 \sqrt{2} \pi^{2} i\left\{[1-\mathcal{M}(++,++)] e^{-i \phi_{1}}\right. \\
& \left.-2 \cos \phi_{1}\right\} .
\end{aligned}
$$

Taking the modulus squared of Eq. (35) and averaging over $\phi_{1}$, we recover Eq. (32) with $\lambda_{2}=\lambda_{3}=\lambda_{4}=+1$. The residual two cases $\lambda_{3}=-\lambda_{4}= \pm 1$ may be treated similarly.

We also check the CPM limit numerically by temporarily adopting the following simple ansatz for the gluon unPDF:

$$
\Phi_{g}\left(x, t, \mu_{F}\right)=x f_{g}\left(x, \mu_{F}\right) \frac{2}{\mu_{F}^{2} \sigma \sqrt{\pi}} \exp \left(-\frac{t^{2}}{\mu_{F}^{4} \sigma^{2}}\right),
$$

with a sufficiently small value of $\sigma$. In fact, $\sigma \rightarrow 0$ corresponds to the CPM limit, in which the normalization condition of Eq. (2) is satisfied.

Finally, we recover our result for Eq. (19), including its full $t_{1}$ dependence, from the vector parts of the helicity amplitudes of the partonic subprocess $g g \rightarrow Z g$ presented in Ref. [33]. To this end, we represent the projector $\left(n_{-}\right)_{\mu_{1}}$ in Eq. (19) as a linear combination of the transverse and longitudinal polarization four-vectors of the $Z$ boson and perform a boost to the center-of-mass frame used in Ref. [33].

\section{NUMERICAL ANALYSIS}

We are now in a position to present our numerical results for the cross section of prompt-photon plus jet associated photoproduction in the PRA and to compare them with 
TABLE I. Kinematic conditions of the HERA II data sets $[1,2,4,5]$.

\begin{tabular}{lcccc}
\hline \hline $\mathrm{H} 1-2005[1]$ & $\mathrm{H} 1-2010[2]$ & ZEUS-2007 I [4] & ZEUS-2007 II [4] & ZEUS-2013 [5] \\
\hline $5.0 \mathrm{GeV}<E_{T}^{\gamma}$ & $6.0 \mathrm{GeV}<E_{T}^{\gamma}$ & $5.0 \mathrm{GeV}<E_{T}^{\gamma}$ & $7.0 \mathrm{GeV}<E_{T}^{\gamma}$ & $6.0 \mathrm{GeV}<E_{T}^{\gamma}$ \\
$<10.0 \mathrm{GeV}$ & $<15.0 \mathrm{GeV}$ & $<16.0 \mathrm{GeV}$ & $<16.0 \mathrm{GeV}$ & $<15.0 \mathrm{GeV}$ \\
$-1.0<\eta^{\gamma}<0.9$ & $-1.0<\eta^{\gamma}<2.4$ & $-0.74<\eta^{\gamma}<1.1$ & $-0.74<\eta^{\gamma}<1.1$ & $-0.7<\eta^{\gamma}<0.9$ \\
$E_{T}^{\text {jet }}>4.5 \mathrm{GeV}$ & $E_{T}^{\text {jet }}>4.5 \mathrm{GeV}$ & $6.0 \mathrm{GeV}<E_{T}^{\text {jet }}<17.0 \mathrm{GeV}$ & $6.0 \mathrm{GeV}<E_{T}^{\text {jet }}<17.0 \mathrm{GeV}$ & $4.0 \mathrm{GeV}<E_{T}^{\text {jet }}<35.0 \mathrm{GeV}$ \\
$-1.0<\eta^{\text {jet }}<2.3$ & $-1.3<\eta^{\text {jet }}<2.3$ & $-1.6<\eta^{\text {jet }}<2.4$ & $-1.6<\eta^{\text {jet }}<2.4$ & $-1.5<\eta^{\text {jet }}<1.8$ \\
$0.2<y<0.7$ & $0.1<y<0.7$ & $0.2<y<0.8$ & $0.2<y<0.8$ & $0.2<y<0.7$ \\
\hline \hline
\end{tabular}

HERA II data $[1,2,4,5]$. We work in the laboratory frame, where the proton and electron have energies $E_{p}=920 \mathrm{GeV}$ and $E_{e}=27.6 \mathrm{GeV}$, respectively, and count rapidity positive in the proton flight direction. We call the transverse energies of the prompt photon and jet $E_{T}^{\gamma}$ and $E_{T}^{\mathrm{jet}}$, their pseudorapidities $\eta^{\gamma}$ and $\eta^{\text {jet }}$, and their azimuthal angles $\phi^{\gamma}$ and $\phi^{\text {jet }}$, respectively. For the reader's convenience, we list our master formula for the hadronic cross section differential
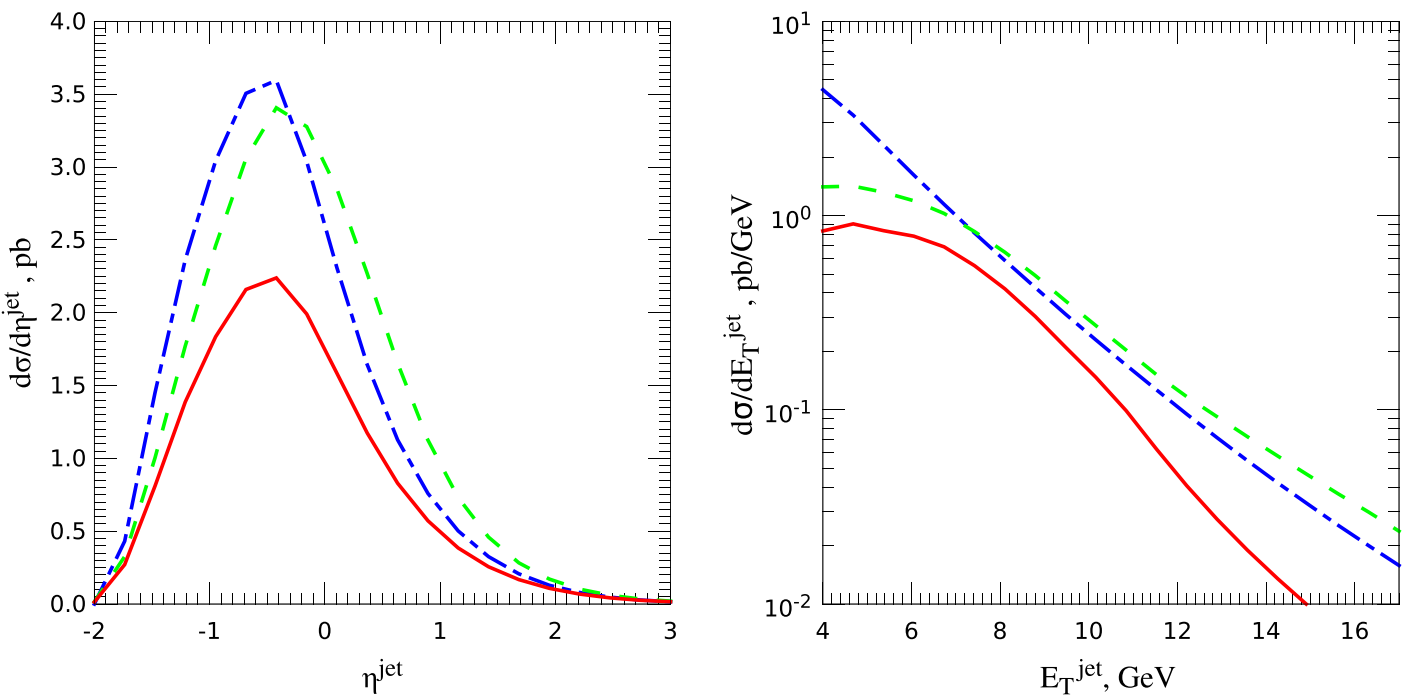

FIG. 5 (color online). Contributions due to the loop-induced subprocess in Eq. (5) to the $\eta^{\text {jet }}$ (left panel) and $E_{T}^{\text {jet }}$ (right panel) distributions of $p e \rightarrow \gamma+j+X$ under H1-2005 [1] kinematic conditions. The exact PRA results (solid red lines) are compared with the approximate results obtained by using the CPM box amplitude in the KFA (dashed green lines) and with the CPM results (dot-dashed blue lines).

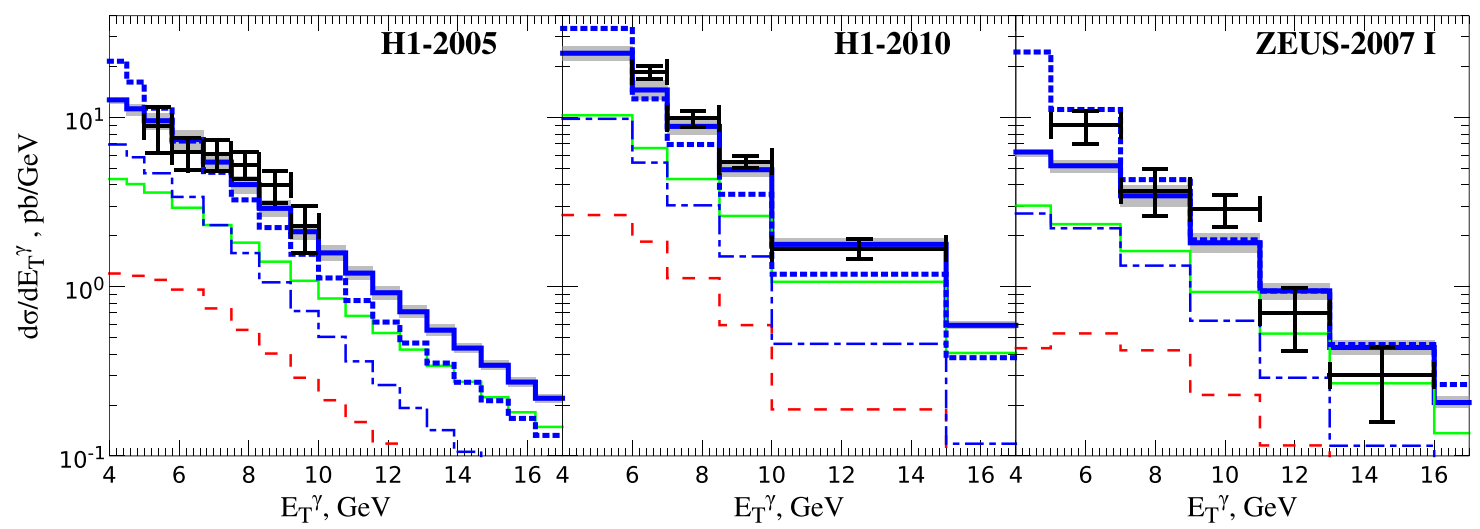

FIG. 6 (color online). $E_{T}^{\gamma}$ distributions of $p e \rightarrow \gamma+j+X$ under H1-2005 [1] (left panel), H1-2010 [2] (central panel), and ZEUS-2007 I [4] (right panel) kinematic conditions. The experimental data are compared with LO PRA (boldfaced solid blue lines) and LO CPM (boldfaced dotted blue lines) predictions. The theoretical errors in the LO PRA predictions due to the freedom in the choice of $\xi$ are indicated by the grey bands. The LO PRA predictions are decomposed into the contributions due to the partonic subprocesses in Eqs. (4) (solid green lines), (5) (dashed red lines), and (6) (dot-dashed blue lines). 


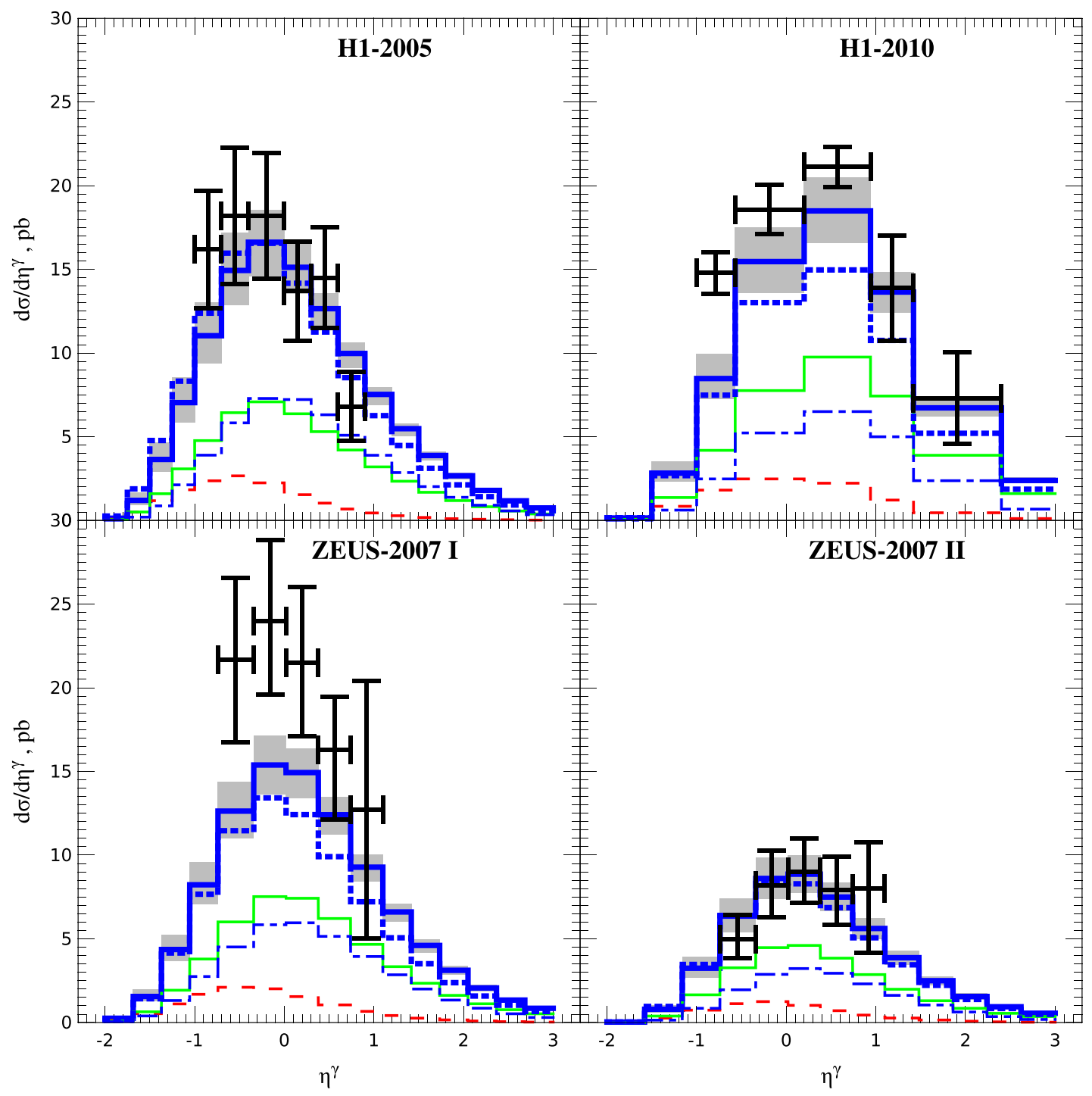

FIG. 7 (color online). $\quad \eta^{\gamma}$ distributions of $p e \rightarrow \gamma+j+X$ under H1-2005 [1] (upper left panel), H1-2010 [2] (upper right panel), ZEUS-2007 I [4] (lower left panel), and ZEUS-2007 II [4] (lower right panel) kinematic conditions. Same notation as in Fig. 6.

in $E_{T}^{\gamma}, \eta^{\gamma}, E_{T}^{\mathrm{jet}}, \eta^{\mathrm{jet}}, \Delta \phi=\phi^{\mathrm{jet}}-\phi^{\gamma}$, and $y$ defined above Eq. (11),

$$
\begin{aligned}
& \frac{d \sigma(p e \rightarrow \gamma+j+X)}{d E_{T}^{\gamma} d \eta^{\gamma} d E_{T}^{\mathrm{jet}} d \eta^{\mathrm{jet}} d(\Delta \phi) d y} \\
& =\sum_{i, j=q, \bar{q}, g} \Phi_{i}\left(x_{1}, t_{1}, \mu_{F}\right) G_{\gamma / e}(y) x_{2} f_{j / \gamma}\left(x_{2}, \mu_{F}\right) \\
& \quad \times \frac{E_{T}^{\gamma} E_{T}^{\mathrm{jet}}}{8 \pi^{2}\left(y x_{1} x_{2} S_{p e}\right)^{2}} \overline{\left|\mathcal{M}_{i j}\right|^{2}},
\end{aligned}
$$

where $S_{p e}=4 E_{p} E_{e}$

$$
\begin{aligned}
& x_{1}=\frac{E_{T}^{\gamma} e^{\eta^{\gamma}}+E_{T}^{\mathrm{jet}} e^{\eta^{\mathrm{jet}}}}{2 E_{p}}, \quad x_{2}=\frac{E_{T}^{\gamma} e^{-\eta^{\gamma}}+E_{T}^{\mathrm{jet}} e^{-\eta^{\mathrm{jet}}}}{2 y E_{e}}, \\
& t_{1}=\left(E_{T}^{\gamma}\right)^{2}+\left(E_{T}^{\mathrm{jet}}\right)^{2}+2 E_{T}^{\gamma} E_{T}^{\mathrm{jet}} \cos (\Delta \phi)
\end{aligned}
$$

In the Weizsäcker-Williams approximation [34], the flux of quasireal photons is

$$
G_{\gamma / e}(y)=\frac{\alpha}{2 \pi}\left[\frac{1+(1-y)^{2}}{y} \ln \frac{Q_{\max }^{2}}{Q_{\min }^{2}}+2 m_{e}^{2} y\left(\frac{1}{Q_{\min }^{2}}-\frac{1}{Q_{\max }^{2}}\right)\right],
$$

where $m_{e}$ is the electron mass, $Q_{\min }^{2}=m_{e}^{2} y^{2} /(1-y)$ is the minimum value of the photon virtuality allowed by kinematics, and its maximum value $Q_{\min }^{2}$ is determined by the experimental conditions to be $Q_{\min }^{2}=1 \mathrm{GeV}^{2}$ in Refs. $[1,2,4,5]$. In the case of resolved photoproduction, $f_{j / \gamma}\left(x_{2}, \mu_{F}\right)$ is the CPM PDF of parton $j$ inside the photon. The case of direct photoproduction is recovered from Eq. (37) by setting $f_{j / \gamma}\left(x_{2}, \mu_{F}\right)=\delta_{\gamma j} \delta\left(1-x_{2}\right)$ and integrating over $x_{2}$ using $d y=-y / x_{2} d x_{2}$, which follows from the second equality of Eq. (38). 


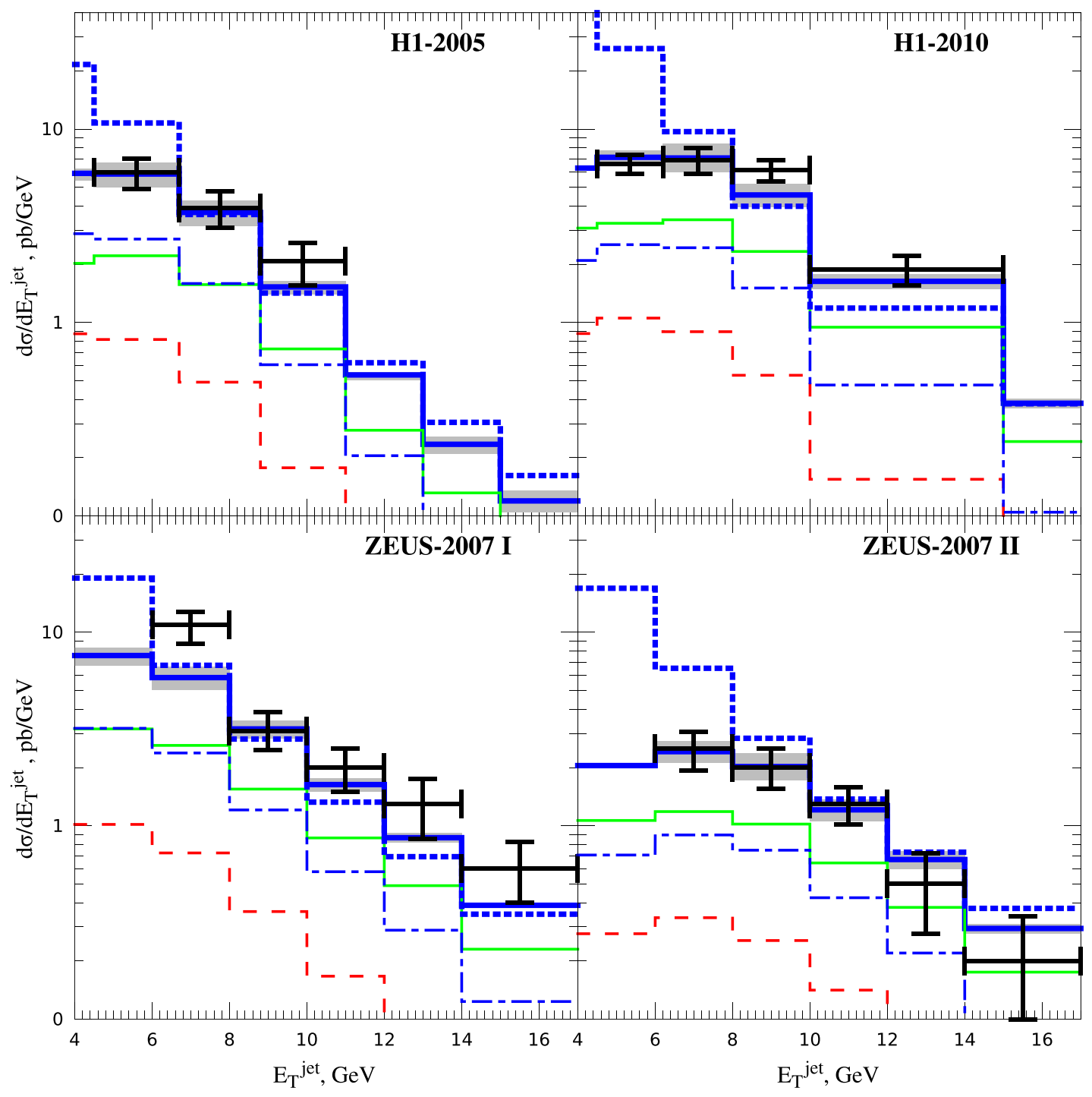

FIG. 8 (color online). $\quad E_{T}^{\mathrm{jet}}$ distributions of $p e \rightarrow \gamma+j+X$ under H1-2005 [1] (upper left panel), H1-2010 [2] (upper right panel), ZEUS-2007 I [4] (lower left panel), and ZEUS-2007 II [4] (lower right panel) kinematic conditions. Same notation as in Fig. 6.

Besides the cross section distributions in $E_{T}^{\gamma}, \eta^{\gamma}, E_{T}^{\mathrm{jet}}, \eta^{\mathrm{jet}}$, $\Delta \phi$, and $y$ given by Eq. (37), also other distributions are measured experimentally. Specifically, the H1 Collaboration also consider the magnitude of the photon's transverse momentum component orthogonal to the direction of the jet transverse momentum $p_{\perp}=E_{T}^{\gamma}|\sin (\Delta \phi)|$. The respective distribution may be obtained from Eq. (37) via the replacement $d E_{T}^{\gamma}=d p_{\perp} /|\sin (\Delta \phi)|$. They also employ the variables

$$
x_{p}^{\mathrm{LO}}=\frac{E_{T}^{\gamma}}{2 E_{p}}\left(e^{\eta^{\gamma}}+e^{\eta^{\mathrm{jet}}}\right), \quad x_{\gamma}^{\mathrm{LO}}=\frac{E_{T}^{\gamma}}{2 y E_{e}}\left(e^{-\eta^{\gamma}}+e^{-\eta^{\mathrm{jet}}}\right),
$$

which, at LO in the CPM, coincide with the fractions of the proton and photon momentum transferred to the initial-state partons. The respective distributions follow from Eq. (37) via the substitutions $d E_{T}^{\gamma}=E_{T}^{\gamma} / x_{p}^{\mathrm{LO}} d x_{p}^{\mathrm{LO}}$ and $d E_{T}^{\gamma}=E_{T}^{\gamma} / x_{\gamma}^{\mathrm{LO}} d x_{\gamma}^{\mathrm{LO}}, \quad$ respectively. The ZEUS Collaboration uses an alternative variable to probe the longitudinal-momentum fraction of the parton in the resolved photon, namely $x_{\gamma}^{\text {obs }}=x_{2}$, where $x_{2}$ is given by the second equality in Eq. (38). The respective distribution emerges from Eq. (37) via the replacement $d E_{T}^{\gamma}=2 y E_{e} e^{\eta^{\gamma}} d x_{\gamma}^{\text {obs }}$. Directphotoproduction subprocesses at LO in the PRA yield contributions proportional to $\delta\left(1-x_{\gamma}^{\text {obs }}\right)$, which are smeared out only by nonfactorizable NLO corrections.

As inputs we use $\alpha=1 / 137.036$, the LO formula for $\alpha_{s}^{\left(n_{f}\right)}\left(\mu_{R}\right)$ with $\Lambda_{\mathrm{LO}}^{\left(n_{f}\right)}=220 \mathrm{MeV}$ for $n_{f}=4$ active quark flavors [35], the proton unPDF set derived from the LO proton PDF set by Martin et al. [35] with $n_{f}=4$ as explained in Ref. [15], and the LO photon PDF set by Glück et al. [36] unless otherwise stated. To estimate the uncertainty related to the photon PDFs, we also use the sets 


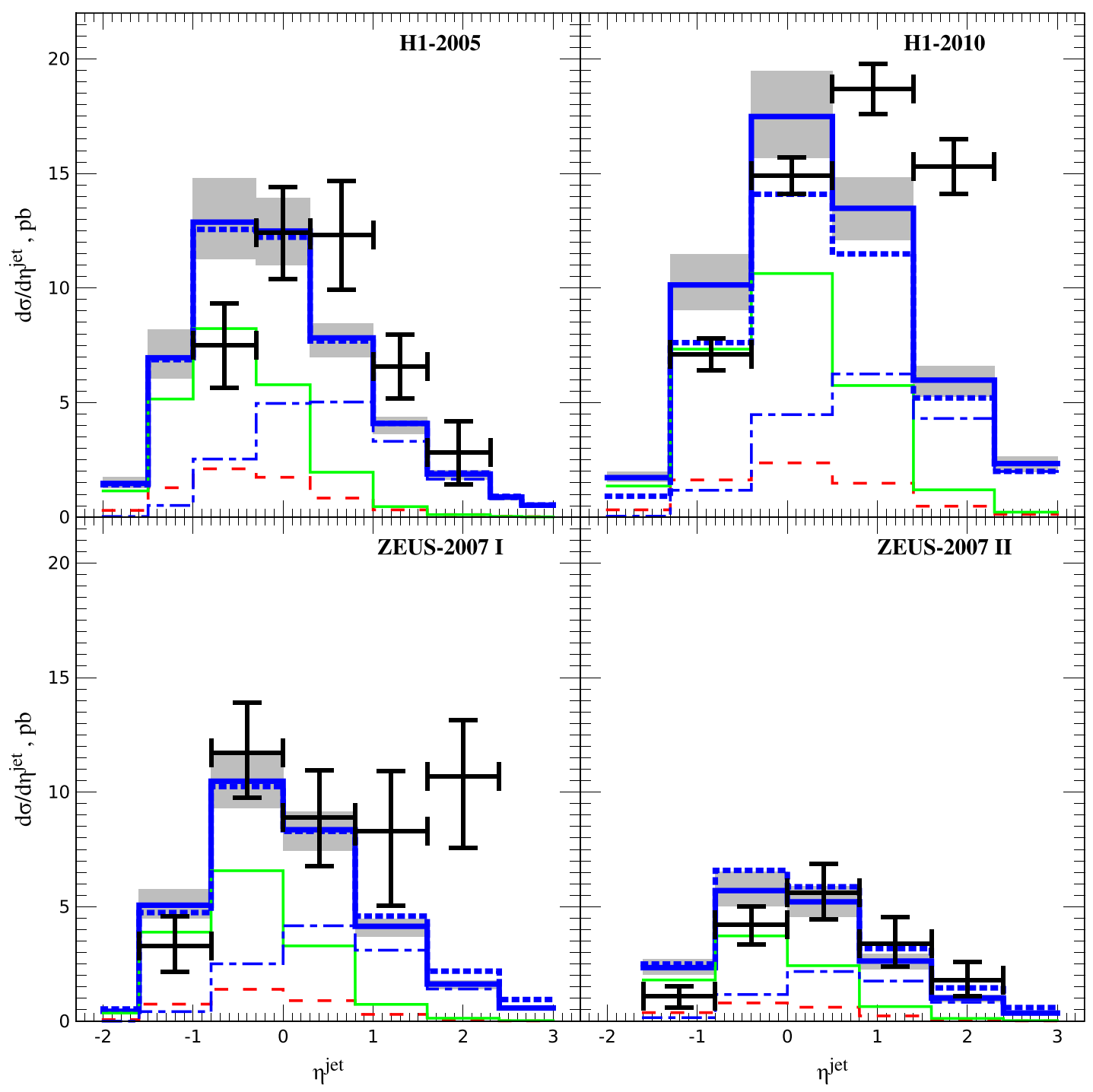

FIG. 9 (color online). $\quad \eta^{\text {jet }}$ distributions of $p e \rightarrow \gamma+j+X$ under H1-2005 [1] (upper left panel), H1-2010 [2] (upper right panel), ZEUS-2007 I [4] (lower left panel), and ZEUS-2007 II [4] (lower right panel) kinematic conditions. Same notation as in Fig. 6.

of Refs. [37-40] as implemented in the PDF library LHAPDF [41]. For our LO CPM predictions, we use the LO proton PDF set [35] mentioned above. We choose the factorization and renormalization scales to be $\mu_{F}=\mu_{R}=$ $\xi \max \left(E_{T}^{\gamma}, E_{T}^{\text {jet }}\right)$ and vary the parameter $\xi$ in the range $1 / 2 \leq$ $\xi \leq 2$ about its default value $\xi=1$.

We compare our results with five experimental data sets collected by the H1 and ZEUS collaborations at HERA II, which we refer to as H1-2005 [1], H1-2010 [2], ZEUS2007 I [4], ZEUS-2007 II [4], and ZEUS-2013 [5]. The respective kinematic conditions are summarized in Table I.

Prior to comparing with experimental data, we assess the significance of rigorously evaluating the loop-induced subprocess in Eq. (5) in the PRA, from Eqs. (37) and (A11), rather than using the CPM box amplitude in the context of the KFA as was done in Ref. [10]. We do this in Fig. 5 for the $\eta^{\text {jet }}$ and $E_{T}^{\text {jet }}$ distributions under H1-2005 [1] kinematic conditions. We observe that, except for small values of $E_{T}^{\mathrm{jet}}$, the approximation of Ref. [10] (dashed green lines) is very close the pure CPM result (dot-dashed blue lines) and significantly overshoots the genuine PRA result (solid red lines), by as much as $50 \%$ at the peak of the $\eta^{\text {jet }}$ distribution.

We now turn to the comparisons with the HERA II data $[1,2,4,5]$. Specifically, we consider the $E_{T}^{\gamma}, \eta^{\gamma}, E_{T}^{\mathrm{jet}}$, and $\eta^{\mathrm{jet}}$ distributions of Refs. [1,2,4] in Figs. 6, 7, 8, and 9, respectively, the same distributions of Ref. [5] in Fig. 10, the $x_{p}^{\mathrm{LO}}$ distributions of Refs. [1,2] in Fig. 11, the $x_{\gamma}^{\mathrm{LO}}$ distributions of Refs. [1,2] and the $x_{\gamma}^{\text {obs }}$ distributions of Ref. [4] in Fig. 12, the $x_{\gamma}^{\text {obs }}$ distribution of Ref. [5] in Fig. 13, the normalized $\Delta \phi$ distributions of Ref. [2] in Fig. 14, and the normalized $p_{\perp}$ distributions of Refs. [1,2] in Fig. 15. In each figure, the LO PRA (boldfaced solid blue lines) 

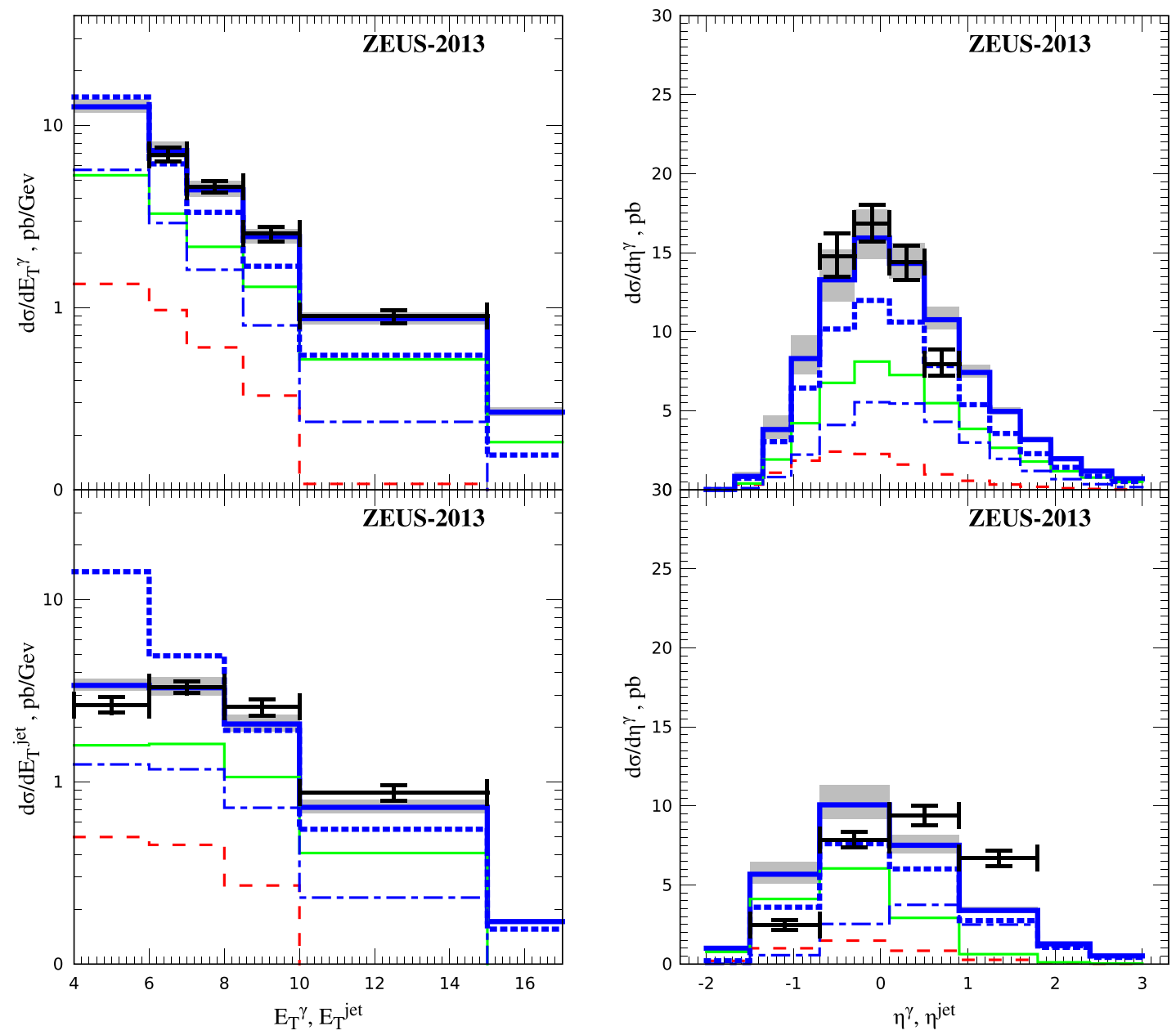

FIG. 10 (color online). $\quad E_{T}^{\gamma}$ (upper left panel), $\eta^{\gamma}$ (upper right panel), $E_{T}^{\text {jet }}$ (lower left panel), and $\eta^{\text {jet }}$ (lower right panel) distributions of $p e \rightarrow \gamma+j+X$ under ZEUS-2013 [5] kinematic conditions. Same notation as in Fig. 6.

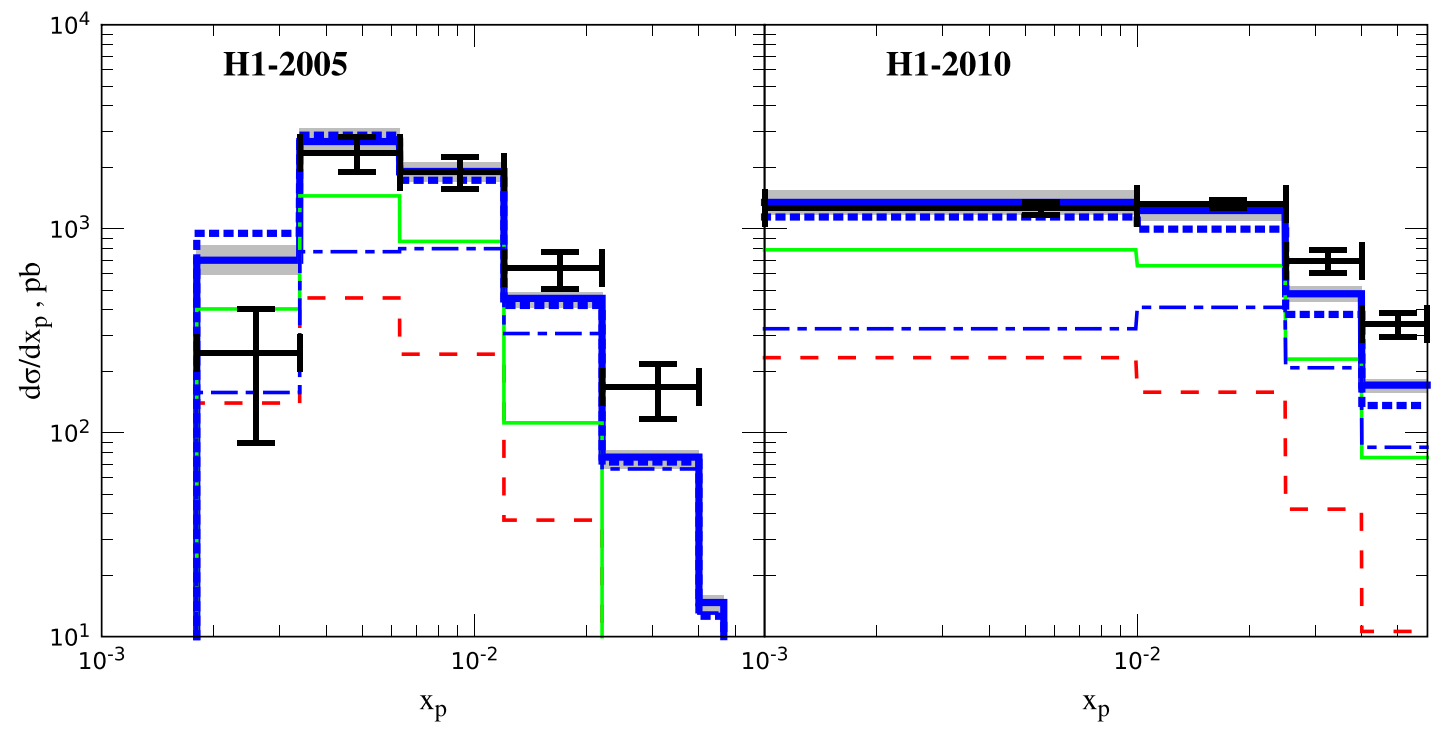

FIG. 11 (color online). $\quad x_{p}^{\mathrm{LO}}$ distributions of $p e \rightarrow \gamma+j+X$ under H1-2005 [1] (left panel) and H1-2010 [2] (right panel) kinematic conditions. Same notation as in Fig. 6. 


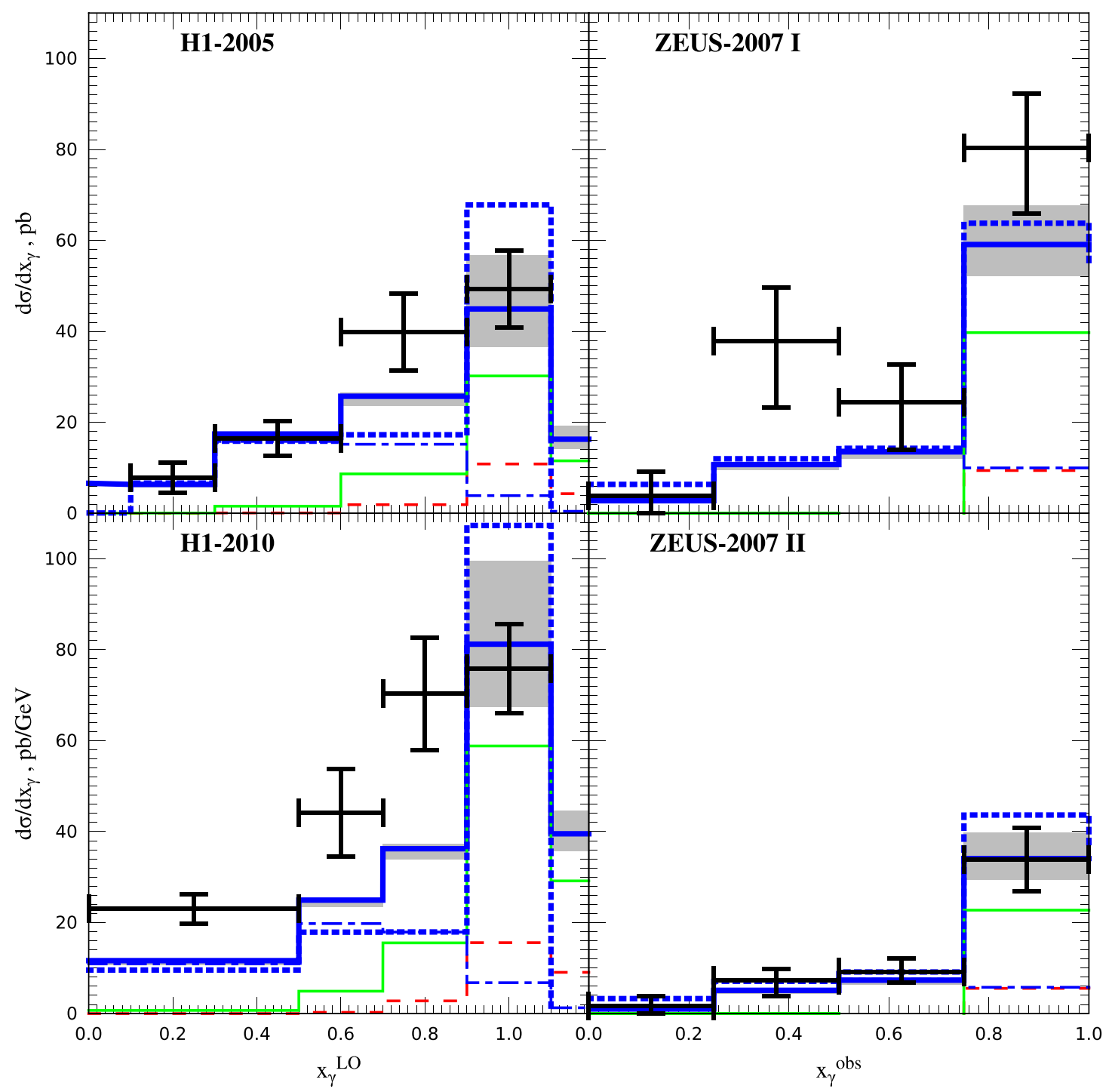

FIG. 12 (color online). $\quad x_{\gamma}^{\mathrm{LO}}$ distributions of $p e \rightarrow \gamma+j+X$ under H1-2005 [1] (upper left panel) and H1-2010 [2] (lower left panel) kinematic conditions and $x_{\gamma}^{\text {obs }}$ distributions of $p e \rightarrow \gamma+j+X$ under ZEUS-2007 I [4] (upper right panel) and ZEUS-2007 II [4] (lower right panel) kinematic conditions. Same notation as in the Fig. 6.

predictions are decomposed into the contributions due to the partonic subprocesses in Eqs. (4) (solid green lines), (5) (dashed red lines), and (6) (dot-dashed blue lines) and compared with the LO CPM predictions (boldfaced dotted blue lines). The theoretical errors in the LO PRA predictions due to the freedom in the choice of $\xi$ are indicated by the grey bands. The normalization factors $\sigma$ in Figs. 14 and 15 are evaluated using the corresponding $x_{\gamma}^{\mathrm{LO}}$ cuts. Comparisons of the experimental data $[1,2,4,5]$ with NLO CPM predictions $[6,7]$ may be found for the $E_{T}^{\gamma}$ distribution in Fig. 4(c) of Ref. [1], in Fig. 7(a) of Ref. [2], in Fig. 5(a) (ZEUS-2007 I) of Ref. [4], and in Fig. 5(a) of Ref. [5]; for the $\eta^{\gamma}$ distribution in Fig. 4(d) of Ref. [1], in Fig. 7(b) of Ref. [2], in Figs. 5(b) (ZEUS-2007 I) and 8(a) (ZEUS-2007 II) of Ref. [4], and in Fig. 5(b) of Ref. [5]; for the $E_{T}^{\text {jet }}$ distribution in Fig. 5(a) of Ref. [1], in Fig. 7(c) of Ref. [2], in Figs. 6(a) (ZEUS-2007 I) and 8(b) (ZEUS-2007 II) of Ref. [4], and in Fig. 6(a) of Ref. [5]; for the $\eta^{\text {jet }}$ distribution in Fig. 5(b) of Ref. [1], in Fig. 7(d) of Ref. [2], in Figs. 6(b) (ZEUS-2007 I) and 8(c) (ZEUS-2007 II) of Ref. [4], and in Fig. 6(b) of Ref. [5]; for the $x_{p}^{\mathrm{LO}}$ distribution in Fig. 5(d) of Ref. [1] and in Fig. 8(b) of Ref. [2]; for the $x_{\gamma}^{\mathrm{LO}}$ or $x_{\gamma}^{\text {obs }}$ distributions in Fig. 5(c) of Ref. [1], in Fig. 8(a) of Ref. [2], in Figs. 7 (ZEUS-2007 I) and 9 (ZEUS-2007 II) of Ref. [4], and in Fig. 7 of Ref. [5]; for the $\Delta \phi$ distribution in Figs. 9(a) $\left(x_{\gamma}^{\mathrm{LO}}>0.8\right)$ and $9(\mathrm{c})\left(x_{\gamma}^{\mathrm{LO}}<0.8\right)$ of Ref. [2], and for the $p_{\perp}$ distribution in Figs. 6(c) $\left(x_{\gamma}^{\mathrm{LO}}<0.85\right)$ and $6(\mathrm{~d})\left(x_{\gamma}^{\mathrm{LO}}>0.85\right)$ of Ref. [1] and in Figs. 9(b) $\left(x_{\gamma}^{\mathrm{LO}}>0.8\right)$ and 9(d) $\left(x_{\gamma}^{\mathrm{LO}}<0.8\right)$ in Ref. [2].

We first assess the relative importance of the LO PRA contributions due to the partonic subprocesses in Eqs. (4)-(6). When $x_{\gamma}^{\mathrm{LO}}$ or $x_{\gamma}^{\mathrm{obs}}$ are not constrained, as in Figs. 6-11, then the partonic subprocesses in Eqs. (4) and 


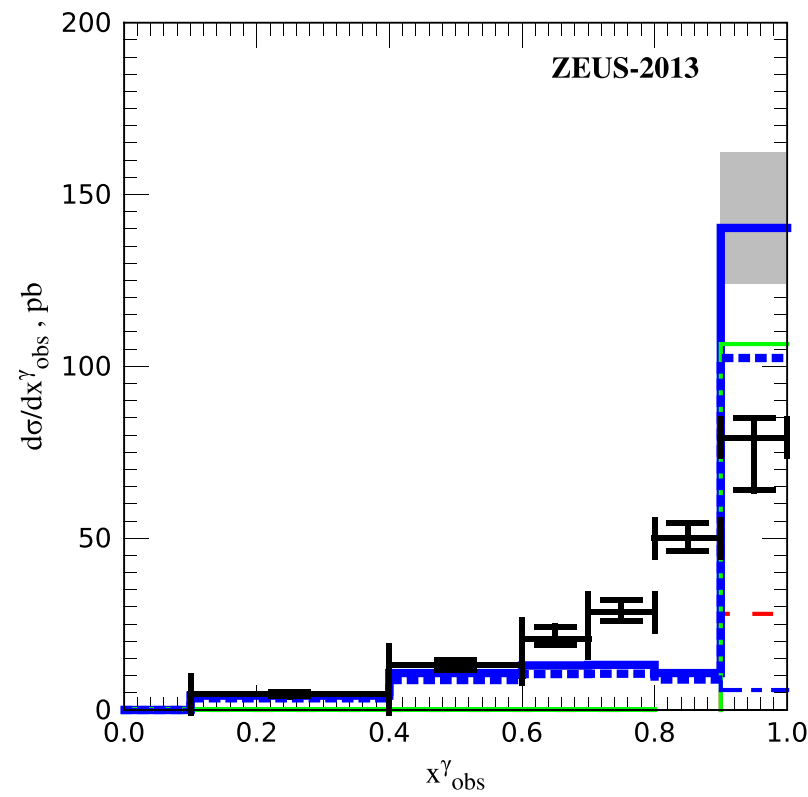

FIG. 13 (color online). $x_{\gamma}^{\mathrm{obs}}$ distribution of $p e \rightarrow \gamma+j+X$ under ZEUS-2013 [5] kinematic conditions. Same notation as in the Fig. 6.

(6) compete with each other, while the one in Eq. (5) is of minor importance. Obviously, the loop suppression of the latter is insufficiently compensated by the dominance of the gluon unPDF over the quark unPDFs. This feature is more pronounced in the PRA than in the CPM or in the approximation of Ref. [10], as we have seen in Fig. 5. Looking at Figs. 12 and 13, we observe that resolved photoproduction, which essentially proceeds via the partonic subprocess in Eq. (6), dominates for $x_{\gamma}^{\mathrm{LO}}<0.9$ after H1-2005 [1] or H1-2010 [2] cuts, for $x_{\gamma}^{\text {obs }}<0.75$ after ZEUS-2007 I [4] and ZEUS-2007 II [4] cuts, and for $x_{\gamma}^{\text {obs }}<0.9$ after ZEUS-2013 [5] cuts. This is also reflected in Figs. 14 and 15, where the LO PRA predictions for $x_{\gamma}^{\mathrm{LO}}<0.85$ [1] and $x_{\gamma}^{\mathrm{LO}}<0.8$ [2] are almost exhausted by the contribution due the partonic subprocess in Eq. (6). By contrast, the partonic subprocesses of direct photoproduction in Eqs. (4) and (5) only contribute to the utmost $x_{\gamma}^{\text {obs }}$ bins in Figs. 12 and 13. In order for this peak to be smeared out, one needs to include $2 \rightarrow 3$ subprocesses of direct photoproduction at NLO giving rise to an additional jet in the central region of rapidity. From the lower left panel in Fig. 12, we observe that the LO PRA prediction typically undershoots the H1-2010 [2] data by a factor of 2 in the range $x_{\gamma}^{\mathrm{LO}}<0.9$. The same undershoot would show up in the left panel of Fig. 14 and in the lower left panel of Fig. 15 if it were not for the normalizations of the $\Delta \phi$ and $p_{\perp}$ distributions shown there.

Next we compare the LO PRA predictions with the LO CPM ones. From Figs. 6, 8, and 10, we observe that the $E_{T}^{\gamma}$ and $E_{T}^{\text {jet }}$ distributions generally fall off more steeply in the CPM and significantly overshoot the PRA distributions at small values of $E_{T}^{\gamma}$ and $E_{T}^{\mathrm{jet}}$. This may be attributed to the fact that the singular behavior of the partonic cross sections for $E_{T}^{\gamma} \rightarrow 0$ or $E_{T}^{\mathrm{jet}} \rightarrow 0$ in the CPM is washed out by the PRA dynamics and the $k_{T}$ smearing via the unPDFs. From Fig. 6, we also learn that the LO CPM predictions for the H1-2005 [1] or H1-2010 [2] experimental conditions undershoot the LO PRA ones for $E_{T}^{\gamma}>6 \mathrm{GeV}$. Consequently, the same is true for the H1-2010 [2] $\eta^{\gamma}$ and $\eta^{\text {jet }}$ distributions in Figs. 7 and 9, respectively, because of the very cut $E_{T}^{\gamma}>6 \mathrm{GeV}$. A similar observation can be made in Fig. 10 for the ZEUS-2013 [5] situation: The LO CPM $E_{T}^{\gamma}$ distribution undershoots the LO PRA one for $E_{T}^{\gamma}>6 \mathrm{GeV}$, which carries over the $\eta^{\gamma}$ and $\eta^{\text {jet }}$ distributions being subject to this very cut. Since the prompt photon and

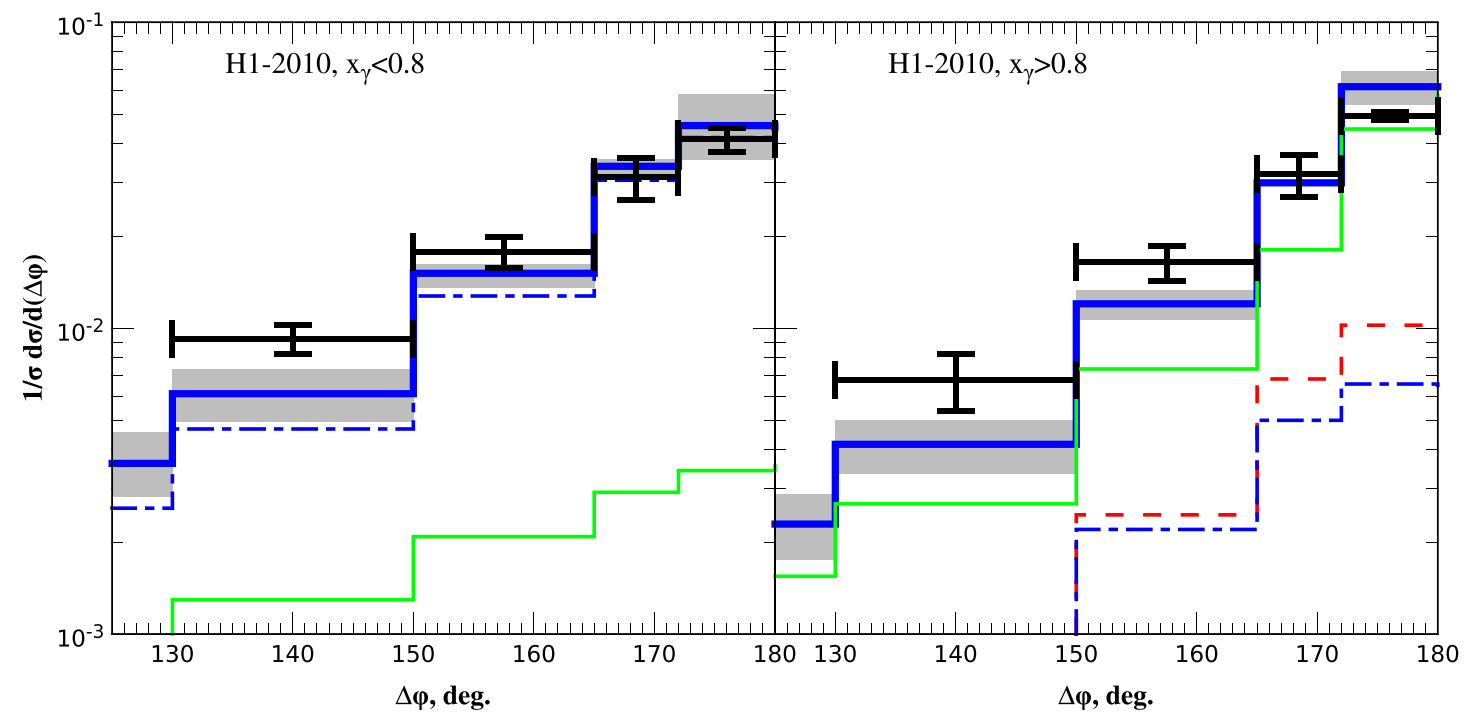

FIG. 14 (color online). Normalized $\Delta \phi$ distributions of $p e \rightarrow \gamma+j+X$ under H1-2010 [2] kinematic conditions for $x_{\gamma}^{\mathrm{LO}}<0.8$ (left panel) and $x_{\gamma}^{\mathrm{LO}}>0.8$ (right panel). Same notation as in the Fig. 6. 


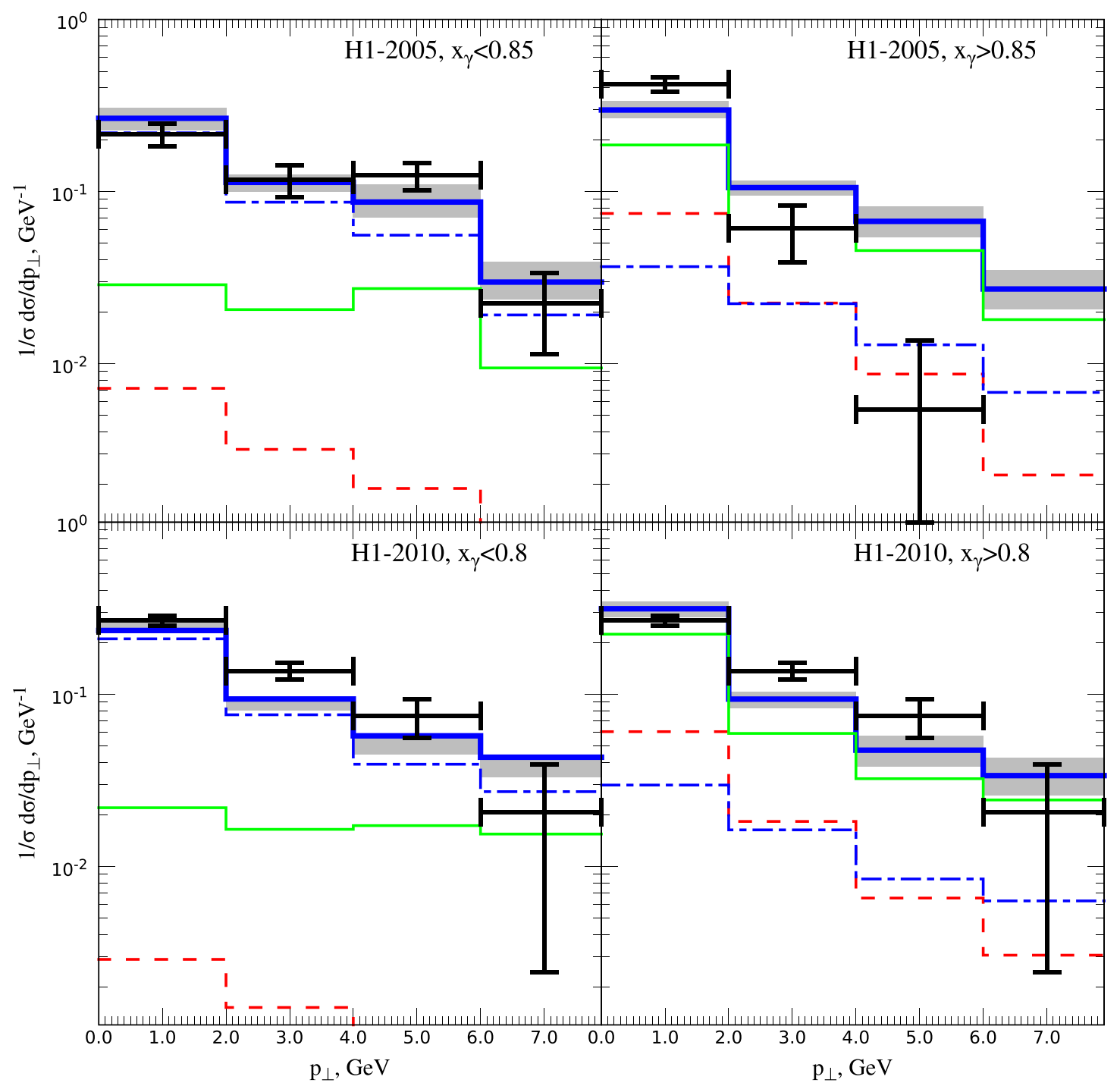

FIG. 15 (color online). Normalized $p_{\perp}$ distributions of $p e \rightarrow \gamma+j+X$ under H1-2005 [1] kinematic conditions for $x_{\gamma}^{\mathrm{LO}}<0.85$ (upper left panel) and $x_{\gamma}^{\mathrm{LO}}>0.85$ (upper right panel) and under H1-2010 [2] kinematic conditions for $x_{\gamma}^{\mathrm{LO}}<0.8$ (lower left panel) and $x_{\gamma}^{\mathrm{LO}}>0.8$ (lower right panel). Same notation as in the Fig. 6.

the jet are strictly back to back at LO in the CPM, the respective contributions to the $\Delta \phi$ and $p_{\perp}$ distributions are zero, as may be seen from Figs. 14 and 15 .

At this point, we estimate the theoretical uncertainty due to the imperfect knowledge of the photon PDFs. We do this by recalculating the $x_{\gamma}^{\mathrm{LO}}$ distributions in Fig. 12, which are particularly sensitive probes of this, using four alternative photon PDF sets [37-40] as implemented in the PDF library LHAPDF [41]. We find the variation to be $\pm(10-20) \%$ in the interval $0.2<x_{\gamma}^{\mathrm{LO}}<0.9$ and below $\pm 10 \%$ in the utmost bin.

Finally, we compare the predictions at LO in the PRA and at NLO in the CPM $[6,7]$ with respect to their abilities to describe the experimental data $[1,2,4,5]$. We find their overall performances to be comparable, except that, at LO in the PRA, the peak positions of the $\eta^{\text {jet }}$ distributions are generally too small and the $x_{p}^{\mathrm{LO}}$ distributions tend to be too small in the utmost bins. On the other hand, the CPM at NLO significantly undershoots the measured $\Delta \phi$ distribution for $x_{\gamma}^{\mathrm{LO}}<0.8$ in the utmost bin, where the PRA at LO does an excellent job. However, these comparisons have to be taken with a grain of salt because the NLO CPM predictions presented in Refs. $[1,2,4,5]$ include corrections due to hadronization and multiple interactions, which are beyond the scope of our present analysis.

\section{CONCLUSIONS}

We studied prompt-photon plus jet associated photoproduction at LO in the PRA, treating the quarks and gluons inside the proton as Reggeized particles and allowing for the incoming photon to be resolved. We also included the loop-induced subprocess in Eq. (5), which was treated in the PRA accounting for the off-shellness of the 
Reggeon in a manifestly gauge-invariant way for the first time. We performed detailed comparisons with experimental data taken by the H1 [1,2] and ZEUS [4,5] collaborations at HERA II, which come as cross section distributions in $E_{T}^{\gamma}, \eta^{\gamma}, E_{T}^{\mathrm{jet}}, \eta^{\mathrm{jet}}, x_{p}^{\mathrm{LO}}, x_{\gamma}^{\mathrm{LO}}, x_{\gamma}^{\mathrm{obs}}, \Delta \phi$, and $p_{\perp}$. We generally found good agreement, which indicates that factorizable higher-order corrections are significant here.

\section{ACKNOWLEDGMENTS}

We thank A. Iudin and K. Nowak for a clarifying communication concerning Ref. [4], E. Lohrmann for drawing our attention to Ref. [5], and A. Kotikov and O. Veretin for useful comments on the box contribution. The work of M. A. N. was supported in part by the German Academic Exchange Service DAAD and the Ministry of Science and Education of the Russian Federation through Michail Lomonosov Grant No. A/12/75163 and by the Dynasty Foundation through a grant from the Graduate Students Stipend Program. The work of M. A. N. and V. A. S. was supported in part by the Russian Foundation for Basic Research through Grant No. 14-02-00021. This work was supported in part by the German Federal Ministry for Education and Research BMBF through Grant No. 05H12GUE.

\section{APPENDIX: BOX AMPLITUDE}

In this appendix, we present the independent helicity amplitudes in Eq. (19) of the partonic subprocess in Eq. (5). They may be written as

$$
\begin{aligned}
& \mathcal{M}(R+,++)=\mathcal{M}\left(t, u, t_{1},\left\{f_{i}^{(1)}\right\}, \mathcal{R}_{1}\right), \\
& \mathcal{M}(R+,+-)=\mathcal{M}\left(s, t, t_{1},\left\{f_{i}^{(2)}\right\}, \mathcal{R}_{2}\right),
\end{aligned}
$$

$$
\begin{gathered}
\mathcal{M}(R+,-+)=\mathcal{M}\left(s, u, t_{1},\left\{f_{i}^{(3)}\right\}, \mathcal{R}_{3}\right), \\
\mathcal{M}(R+,--)=\frac{i \pi^{2} 4 \sqrt{2}}{u \Delta}(t+u) \gamma_{1},
\end{gathered}
$$

where

$$
\begin{aligned}
\mathcal{M}\left(t, u, t_{1},\left\{f_{i}\right\}, \mathcal{R}\right)= & \frac{i \pi^{2}}{\sqrt{2} \Delta^{3}(t+u)}\left\{f_{1}\left[B_{0}(t)-B_{0}\left(-t_{1}\right)\right]\right. \\
& +f_{2}\left[B_{0}(u)-B_{0}\left(-t_{1}\right)\right] \\
& \left.+f_{3} E\left(t_{1}, t, u\right)+\mathcal{R}\right\},
\end{aligned}
$$

with

$$
\begin{aligned}
E\left(t_{1}, t, u\right)= & t C_{0}(t)+u C_{0}(u)+\left(t+t_{1}\right) C_{0}\left(-t_{1}, t\right) \\
& +\left(u+t_{1}\right) C_{0}\left(-t_{1}, u\right)-t u D_{0}\left(-t_{1}, t, u\right) .
\end{aligned}
$$

In the notation of Ref. [32], the scalar one-loop integrals are defined as

$$
\begin{aligned}
B_{0}\left(p_{1}^{2}\right) & =I_{2}^{D}\left(p_{1}^{2} ; 0,0\right), \\
C_{0}\left(p_{3}^{2}\right) & =I_{3}^{D}\left(0,0, p_{3}^{2} ; 0,0,0\right), \\
C_{0}\left(p_{2}^{2}, p_{3}^{2}\right) & =I_{3}^{D}\left(0, p_{2}^{2}, p_{3}^{2} ; 0,0,0\right), \\
D_{0}\left(s_{12}, s_{23}\right) & =I_{4}^{D}\left(0,0,0,0 ; s_{12}, s_{23} ; 0,0,0,0\right), \\
D_{0}\left(p_{4}^{2}, s_{12}, s_{23}\right) & =I_{4}^{D}\left(0,0,0, p_{4}^{2} ; s_{12}, s_{23} ; 0,0,0,0\right) .
\end{aligned}
$$

The coefficients pertaining to Eq. (A1) read

$$
\begin{aligned}
& f_{1}^{(1)}=\frac{-i t^{2}}{2\left(t+t_{1}\right)^{2}}\left\{2(s+2 u)\left(t+t_{1}\right)(t+u)^{2} \gamma_{1}+4 i s u^{2}\left[2 t\left(t+t_{1}\right)-u t_{1}\right] \sqrt{t_{1}}+u\left[s^{2}\left(s+t_{1}\right)+3 s u\left(s-t_{1}\right)+2 u^{2}\left(s-t_{1}\right)\right] i \gamma_{2}\right\}, \\
& f_{2}^{(1)}=\frac{-i t u}{2\left(u+t_{1}\right)^{2}}\left\{2(s+2 t)\left(u+t_{1}\right)(t+u)^{2} \gamma_{1}+4 i s t u\left[t t_{1}-2 u\left(u+t_{1}\right)\right] \sqrt{t_{1}}+u\left[s^{3}+s^{2}\left(3 t+t_{1}\right)+s t\left(2 t-3 t_{1}\right)-2 t^{2} t_{1}\right] i \gamma_{2}\right\}, \\
& f_{3}^{(1)}=\frac{-i t}{4 s}\left\{2(t+u)^{2}\left[t^{2}+t_{1} t+u\left(u+t_{1}\right)\right] \gamma_{1}+4 i s t u^{2}(u-t) \sqrt{t_{1}}+u\left[t^{3}+t^{2}\left(u+t_{1}\right)+t u\left(u-2 t_{1}\right)+u^{2}\left(u+t_{1}\right)\right] i \gamma_{2}\right\}, \\
& \mathcal{R}_{1}=\frac{s t^{2} u^{2}}{\left(t+t_{1}\right)\left(u+t_{1}\right)}\left[\left(t_{1}-s\right) \gamma_{2}+2 s(t-u) \sqrt{t_{1}},\right.
\end{aligned}
$$

where $\gamma_{1}$ and $\gamma_{2}$ are defined in Eqs. (27) and (30), respectively. The coefficients pertaining to Eq. (A2) read

$$
\begin{aligned}
f_{1}^{(2)} & =\frac{-i s^{2} t}{2 u}\left[2(t+u)(2 t+u) \gamma_{1}-4 i t u^{2} \sqrt{t_{1}}-u(2 t+u) i \gamma_{2}\right], \\
f_{2}^{(2)} & =\frac{i s t^{2}}{2 u\left(t+t_{1}\right)^{2}}\left\{2(2 s+u)\left(t+t_{1}\right)(t+u)^{2} \gamma_{1}-4 i s u^{2}\left[u t_{1}+t\left(t+t_{1}\right)\right] \sqrt{t_{1}}-u\left[2\left(s+t_{1}\right) s^{2}+3 s u\left(s+t_{1}\right)+u^{2}\left(s-t_{1}\right)\right] i \gamma_{2}\right\} \\
f_{3}^{(2)} & =\frac{i s t}{4 u^{2}}\left\{2\left[s^{2}+t_{1} s+t\left(t+t_{1}\right)\right](t+u)^{2} \gamma_{1}+4 i s t^{2} u^{2} \sqrt{t_{1}}-u\left[u^{3}+u^{2}\left(3 t+t_{1}\right)+t u\left(4 t+t_{1}\right)+2 t^{2}\left(t+t_{1}\right)\right] i \gamma_{2}\right\} \\
\mathcal{R}_{2} & =-\frac{s^{2} t^{2} u}{t+t_{1}}\left(2 u \sqrt{t_{1}}+\gamma_{2}\right) .
\end{aligned}
$$


The coefficients pertaining to Eq. (A3) emerge from Eq. (A9) via the substitutions

$$
t \leftrightarrow u, \quad \sqrt{t_{1}} \rightarrow-\sqrt{t_{1}}, \quad \gamma_{1} \rightarrow \gamma_{1} \frac{t}{u},
$$

which amounts to permutating the final-state partons.

The modulus square of the hard-scattering amplitude of the partonic subprocess in Eq. (5) averaged over the spins and colors in the initial state and summed over those in the final state is then obtained from the helicity amplitudes in Eqs. (A1)-(A4) as

$$
\overline{|\mathcal{M}(R+\gamma \rightarrow g+\gamma)|^{2}}=\frac{\alpha^{2} \alpha_{s}^{2}}{4 \pi^{4}}\left(\sum_{q} e_{q}^{2}\right)^{2} \sum_{\lambda_{3}, \lambda_{4}= \pm 1}\left|\mathcal{M}\left(R+, \lambda_{3} \lambda_{4}\right)\right|^{2} .
$$

For completeness, we also present the corresponding CPM result [31],

$$
\begin{aligned}
\overline{|\mathcal{M}(g \gamma \rightarrow g \gamma)|^{2}}= & 8 \alpha^{2} \alpha_{s}^{2}\left(\sum_{q} e_{q}^{2}\right)^{2}\left\{|\mathcal{M}(++,++)|^{2}+|\mathcal{M}(-+,-+)|^{2}+|\mathcal{M}(-+,+-)|^{2}+|\mathcal{M}(++,--)|^{2}\right. \\
& \left.+4|\mathcal{M}(++,+-)|^{2}\right\},
\end{aligned}
$$

where

$$
\begin{aligned}
& \mathcal{M}(++,--)=\mathcal{M}(++,+-)=-1, \\
& \mathcal{M}(++,++)=1+(2 x-1) L_{2}+\frac{1}{2}\left[x^{2}+(1-x)^{2}\right]\left(L_{2}+\pi^{2}\right), \\
& \mathcal{M}(-+,-+)=1+\left(1-\frac{2}{x}\right)\left(L_{1}-\pi i\right)+\frac{1}{2 x^{2}}\left[1+(1-x)^{2}\right] L_{1}\left(L_{1}-2 \pi i\right), \\
& \mathcal{M}(-+,+-)=\left.\mathcal{M}(-+,-+)\right|_{x \rightarrow 1-x},
\end{aligned}
$$

with $L_{1}=\log [1 /(1-x)], L_{2}=\log [(1-x) / x]$, and $x=-t / s+i 0$.

[1] A. Aktas et al. (H1 Collaboration), Eur. Phys. J. C 38, 437 (2005).

[2] F. D. Aaron et al. (H1 Collaboration), Eur. Phys. J. C 66, 17 (2010).

[3] J. Breitweg et al. (ZEUS Collaboration), Phys. Lett. B 472, 175 (2000).

[4] S. Chekanov et al. (ZEUS Collaboration), Eur. Phys. J. C 49, 511 (2007).

[5] H. Abramowicz et al. (ZEUS Collaboration), Phys. Lett. B 730, 293 (2014).

[6] M. Fontannaz, J. Ph. Guillet, and G. Heinrich, Eur. Phys. J. C 21, 303 (2001); M. Fontannaz and G. Heinrich, Eur. Phys. J. C 34, 191 (2004).

[7] A. Zembrzuski and M. Krawczyk, Phys. Rev. D 64, 114017 (2001); Report No. IFT-2003-27.

[8] A. V. Lipatov and N. P. Zotov, Phys. Rev. D 72, 054002 (2005).

[9] A. V. Lipatov and N. P. Zotov, Phys. Rev. D 81, 094027 (2010).
[10] A. V. Lipatov, M. A. Malyshev, and N. P. Zotov, Phys. Rev. D 88, 074001 (2013).

[11] V. A. Saleev, Phys. Rev. D 78, 114031 (2008).

[12] L. V. Gribov, E. M. Levin, and M. G. Ryskin, Phys. Rep. 100, 1 (1983); J. C. Collins and R. K. Ellis, Nucl. Phys. B360, 3 (1991); S. Catani, M. Ciafaloni, and F. Hautmann, Nucl. Phys. B366, 135 (1991).

[13] V. N. Gribov and L. N. Lipatov, Yad. Fiz. 15, 781 (1972) [Sov. J. Nucl. Phys. 15, 438 (1972)]; Yu. L. Dokshitser, Zh. Eksp. Teor. Fiz. 73, 1216 (1977) [Sov. Phys. JETP 46, 641 (1977)]; G. Altarelli and G. Parisi, Nucl. Phys. B126, 298 (1977).

[14] L. N. Lipatov, Yad. Fiz. 23, 642 (1976) [Sov. J. Nucl. Phys. 23, 338 (1976)]; É. A. Kuraev, L. N. Lipatov, and V.S. Fadin, Zh. Eksp. Teor. Fiz. 71, 840 (1976) [Sov. Phys. JETP 44, 443 (1976)]; Zh. Eksp. Teor. Fiz. 72, 377 (1977) [Sov. Phys. JETP 45, 199 (1977)]; Ya. Ya. Balitskiŭ and L. N. Lipatov, Yad. Fiz. 28, 1597 (1978) [Sov. J. Nucl. Phys. 28, 822 (1978)]; L. N. Lipatov, Zh. Eksp. Teor. Fiz. 90, 1536 (1986) [Sov. Phys. JETP 63, 904 (1986)]. 
[15] M. A. Kimber, A. D. Martin, and M. G. Ryskin, Eur. Phys. J. C 12, 655 (2000); Phys. Rev. D 63, 114027 (2001); G. Watt, A. D. Martin, and M. G. Ryskin, Eur. Phys. J. C 31, 73 (2003); Phys. Rev. D 70, 014012 (2004); 70, 079902(E) (2004).

[16] M. Ciafaloni, Nucl. Phys. B296, 49 (1988); S. Catani, F. Fiorani, and G. Marchesini, Nucl. Phys. B336, 18 (1990); Phys. Lett. B 234, 339 (1990); G. Marchesini, Nucl. Phys. B445, 49 (1995).

[17] V. S. Fadin and L. N. Lipatov, Nucl. Phys. B406, 259 (1993); B477, 767 (1996).

[18] L. N. Lipatov, Phys. Rep. 286, 131 (1997).

[19] L. N. Lipatov, Nucl. Phys. B452, 369 (1995).

[20] L. N. Lipatov and M. I. Vyazovsky, Nucl. Phys. B597, 399 (2001).

[21] E. N. Antonov, I. O. Cherednikov, E. A. Kuraev, and L. N. Lipatov, Nucl. Phys. B721, 111 (2005).

[22] V. A. Saleev and D. V. Vasin, Phys. Lett. B 605, 311 (2005).

[23] B. A. Kniehl, D. V. Vasin, and V. A. Saleev, Phys. Rev. D 73, 074022 (2006); B. A. Kniehl, D. V. Vasin, and V. A. Saleev in Proceedings of the 15th International Workshop on Deep-Inelastic Scattering and Related Subjects (DIS 2007), Munich, Germany, 2007, edited by G. Grindhammer and K. Sachs (Verlag Deutsches Elektronen-Synchrotron, Hamburg, 2007), p. 915.

[24] B. A. Kniehl, V. A. Saleev, and D. V. Vasin, Phys. Rev. D 74, 014024 (2006).

[25] M. A. Nefedov, V. A. Saleev, and A. V. Shipilova, Phys. Rev. D 87, 094030 (2013).

[26] V. A. Saleev and A. V. Shipilova, Phys. Rev. D 86, 034032 (2012).

[27] M. A. Nefedov, N. N. Nikolaev, and V. A. Saleev, Phys. Rev. D 87, 014022 (2013).
[28] B. A. Kniehl, V. A. Saleev, A. V. Shipilova, and E. V. Yatsenko, Phys. Rev. D 84, 074017 (2011).

[29] M. Hentschinski and A. Sabio Vera, Phys. Rev. D 85, 056006 (2012).

[30] A. van Hameren, K. Kutak, and T. Salwa, Phys. Lett. B 727, 226 (2013).

[31] V. Costantini, B. De Tollis, and G. Pistoni, Nuovo Cimento Soc. Ital. Fis. 2A, 733 (1971); V. N. Baier, V. S. Fadin, V. M. Katkov, and E. A. Kuraev, Phys. Lett. 49B, 385 (1974).

[32] R. K. Ellis and G. Zanderighi, J. High Energy Phys. 02 (2008) 002.

[33] J. J. van der Bij and E. W. N. Glover, Nucl. Phys. B313, 237 (1989); E. W. N. Glover and J. J. van der Bij, Nucl. Phys. B321, 561 (1989).

[34] E. J. Williams, Proc. R. Soc. Lond. A 139, 163 (1933); C. F. v. Weizsäcker, Z. Phys. 88, 612 (1934); V. M. Budnev, I. F. Ginzburg, G. V. Meledin, and V. G. Serbo, Phys. Rep. 15, 181 (1975).

[35] A. D. Martin, W. J. Stirling, and R. S. Thorne, Phys. Lett. B 636, 259 (2006).

[36] M. Glück, E. Reya, and A. Vogt, Phys. Rev. D 46, 1973 (1992).

[37] D. W. Duke and J.F. Owens, Phys. Rev. D 26, 1600 (1982).

[38] H. Abramowicz, K. Charchula, and A. Levy, Phys. Lett. B 269, 458 (1991).

[39] G. A. Schuler and T. Sjöstrand, Z. Phys. C 68, 607 (1995).

[40] G. A. Schuler and T. Sjöstrand, Phys. Lett. B 376, 193 (1996).

[41] M. R. Whalley, D. Bourilkov, and R. C. Group, in Proceedings of HERA and the LHC: A Workshop for the Implications of HERA for LHC Physics, edited by A. De Roeck and H. Jung (Report Nos. CERN-2005-014 and DESYPROC-2005-00); https://lhapdf.hepforge.org/. 